\title{
A Characterization of Weighted Carleson Measure Spaces
}

\author{
Hsun-Wu Liu* and Kunchuan Wang
}

\begin{abstract}
Using Frazier and Jawerth's $\varphi$-transform, we characterize weighted generalized Carleson measure spaces $\dot{C} M O_{p, w}^{\alpha, q}$ for a weight $w$ and show that the definition of this space is well-defined by a Plancherel-Pôlya inequality. Note that $\dot{C} M O_{1, w}^{0,2}$ is the weighted $B M O$ space.
\end{abstract}

\section{Introduction}

The general Triebel-Lizorkin spaces $\dot{F}_{p}^{\alpha, q}$ (homogeneous) and $F_{p}^{\alpha, q}$ (inhomogeneous), $\alpha \in$ $\mathbb{R}, 0<p, q \leq \infty$, include many well-known classical function spaces. For example, $L^{p} \approx$ $\dot{F}_{p}^{0,2} \approx F_{p}^{0,2}$ when $1<p<\infty, \dot{F}_{p}^{\alpha, 2} \approx \dot{L}_{p}^{\alpha}$ and $F_{p}^{\alpha, 2} \approx L_{p}^{\alpha}$ (Sobolev spaces) when $1<p<\infty$ and $\alpha>0, H^{p} \approx \dot{F}_{p}^{0,2}$ when $0<p \leq 1$, and $B M O \approx \dot{F}_{\infty}^{0,2}$. Here the notation " $\approx$ " means in a (quasi-)normed vector space $V$ with different norms $\|\cdot\|_{a}$ and $\|\cdot\|_{b}$, there exist positive constants $c_{1}$ and $c_{2}$ such that

$$
c_{1}\|x\|_{a} \leq\|x\|_{b} \leq c_{2}\|x\|_{a}
$$

for all $x$ in $V$.

We say that a cube $Q \subseteq \mathbb{R}^{n}$ is dyadic if $Q=Q_{j \boldsymbol{k}}=\left\{x=\left(x_{1}, x_{2}, \ldots, x_{n}\right) \in \mathbb{R}^{n}\right.$ : $\left.2^{-j} k_{i} \leq x_{i}<2^{-j}\left(k_{i}+1\right), i=1,2, \ldots, n\right\}$ for some $j \in \mathbb{Z}$ and $\boldsymbol{k}=\left(k_{1}, k_{2}, \ldots, k_{n}\right) \in \mathbb{Z}^{n}$. For any pair of dyadic cubes $P$ and $Q$, either $P$ and $Q$ are nonoverlapping or one contains the other. Denote by $\ell(Q)=2^{-j}$ the side length of $Q$ and $x_{Q}=2^{-j} \boldsymbol{k}$ the "left lower corner" of $Q$. In fact $Q=x_{Q}+\left[0,2^{-j}\right)^{n}$. For $j \in \mathbb{Z}$ let $\mathscr{Q}_{j}$ be the collection of dyadic cubes with side length $2^{-j}$ and let $\mathscr{Q}$ be the collection of all dyadic cubes in $\mathbb{R}^{n}$. Thus $\mathscr{Q}=\bigcup_{j \in \mathbb{Z}} \mathscr{Q}_{j}$. For a fixed dyadic cube $P$ let $\mathscr{Q}_{P}$ be the collection of all dyadic cubes in $\mathbb{R}^{n}$ which are contained in $P$.

Received October 22, 2017; Accepted April 26, 2018.

Communicated by Xiang Fang.

2010 Mathematics Subject Classification. 42 B35.

Key words and phrases. Carleson measure, Plancherel-Pôlya inequality, Triebel-Lizorkin space, weight. This research was supported by Ministry of Science and Technology, R.O.C. under Grant \#MOST 1052115-M-259-002.

*Corresponding author. 
For any function $f$ defined on $\mathbb{R}^{n}, j \in \mathbb{Z}$ and dyadic cube $Q=Q_{j \boldsymbol{k}}$, set

$$
\begin{aligned}
f_{Q}(x) & =|Q|^{-1 / 2} f\left(\left(x-x_{Q}\right) / \ell(Q)\right)=2^{j n / 2} f\left(2^{j} x-\boldsymbol{k}\right), \\
f_{j}(x) & =2^{j n} f\left(2^{j} x\right), \\
\widetilde{f}(x) & =\overline{f(-x)} .
\end{aligned}
$$

It is clear that $\widetilde{g}_{j} * f\left(x_{Q}\right)=|Q|^{-1 / 2}\left\langle f, g_{Q}\right\rangle$, where $\langle f, g\rangle$ denotes the pairing in the usual sense for $g$ in a Fréchet space $X$ and $f$ in the dual of $X$.

Next, let us consider more general function spaces. For this purpose, let us recall the $\varphi$-transform identity introduced by Frazier and Jawerth [6]. Choose a fixed Schwartz function $\varphi$ satisfying

$$
\operatorname{supp}(\widehat{\varphi}) \subseteq\{\xi: 1 / 2 \leq|\xi| \leq 2\} \quad \text { and } \quad|\widehat{\varphi}(\xi)| \geq c>0 \quad \text { if } 3 / 5 \leq|\xi| \leq 5 / 3,
$$

where $\widehat{f}$ is the Fourier transform of $f$, i.e.,

$$
\widehat{f}(\xi)=\int_{\mathbb{R}^{n}} f(x) e^{-i x \cdot \xi} d x .
$$

The existence of such a function was provided by Frazier and Jawerth in 6 . Then there exists a function $\psi \in \mathscr{S}$ satisfying the same conditions as $\varphi$ such that

$$
\sum_{j \in \mathbb{Z}} \bar{\varphi}\left(2^{-j} \xi\right) \widehat{\psi}\left(2^{-j} \xi\right)=1 \quad \text { for } \xi \neq 0 .
$$

Thus the $\varphi$-transform identity is given by

$$
f=\sum_{Q \in \mathscr{Q}}\left\langle f, \varphi_{Q}\right\rangle \psi_{Q}
$$

where the identity holds in the sense of $\mathscr{S}^{\prime} / \mathscr{P}$ (the spaces of all tempered distributions modulo polynomials), $\mathscr{S}_{0}$ (the subspace of $\mathscr{S}$ that each element has all vanishing moments), $\dot{B}_{p}^{\alpha, q}$-norm, and $\dot{F}_{p}^{\alpha, q}$-norm.

Now due to the Littlewood-Paley characterization, define the homogeneous TriebelLizorkin spaces as follows. Select a function $\varphi \in \mathscr{S}$ satisfying the conditions above in (1.1). For $\alpha \in \mathbb{R}, 0<p, q \leq \infty$, and $f \in \mathscr{S}^{\prime} / \mathscr{P}$, define the homogeneous Triebel-Lizorkin space to be the collection of every distribution $f \in \mathscr{S}^{\prime} / \mathscr{P}$ so that the norm $\|f\|_{\dot{F}_{p}^{\alpha, q}}$ is finite, where

$$
\|f\|_{\dot{F}_{p}^{\alpha, q}}:=\left\|\left[\sum_{\nu \in \mathbb{Z}}\left(2^{\nu \alpha}\left|\varphi_{\nu} * f\right|\right)^{q}\right]^{1 / q}\right\|_{L^{p}}
$$

if $0<p<\infty$ and

$$
\|f\|_{\dot{F}_{\infty}^{\alpha, q}}:=\sup _{P \in \mathscr{Q}}\left\{\frac{1}{|P|} \int_{P} \sum_{k \geq-\log _{2} \ell(P)}\left(2^{k \alpha}\left|\varphi_{k} * f(x)\right|\right)^{q} d x\right\}^{1 / q} .
$$


By an inequality of Plancherel-Plôlya type, the definition of $\dot{F}_{p}^{\alpha, q}$ is independent of the choice of $\varphi$ satisfying conditions 1.1 .

In 2009 M.-Y. Lee, C.-C. Lin, and Y.-C. Lin 9] characterized the weighted Carleson measure space $C M O_{w}^{p}$ for a weight $w$ belonging to the Muckenhoupt class by wavelets. They lifted sequence spaces to prove that $C M O_{w}^{p}$ is the dual space of $H_{w}^{p}$. The weighted Carleson measure space $C M O_{p}^{w}$ is the set of all $g \in L_{\mathrm{loc}}^{1}$ satisfying

$$
\|g\|_{C M O_{w}^{p}}:=\sup _{J \in \mathscr{D}}\left\{w(J)^{1-2 / p} \sum_{I \in \mathscr{D}_{J}} \frac{|I|}{w(I)}\left|\left\langle g, \psi_{I}\right\rangle\right|^{2}\right\}^{1 / 2}<\infty, \quad 0<p \leq 1,
$$

where $w \in A_{\infty}$ (see Definition 2.2 for the $A_{p}$ weights), $\psi$ is a certain smooth function so that it is an orthonormal wavelet in $L^{2}(w), \mathscr{D}$ is the set of all dyadic intervals $I_{j, k}$ with $j, k \in \mathbb{Z}$, and $\mathscr{D}_{J}$ is the collection of all dyadic intervals contained in $J$.

In 2012, C.-C. Lin and K. Wang [10] gave another characterization for the dual of $\dot{F}_{p}^{\alpha, q}$ in terms of Carleson measures for $\alpha \in \mathbb{R}$ and $0<p \leq 1 \leq q \leq \infty$. The generalized Carleson measure space $C M O_{p}^{\alpha, q}$ is the collection of all $f \in \mathscr{S}^{\prime} / \mathscr{P}$ satisfying $\|f\|_{C M O_{p}^{\alpha, q}}<\infty$, where

$$
\mid f \|_{C M O_{p}^{\alpha, q}}:=\sup _{P \in \mathscr{Q}}\left\{|P|^{-q\left(1 / p-1 / q^{\prime}\right)} \int_{P} \sum_{Q \in \mathscr{Q}_{P}}\left(|Q|^{-\alpha / n-1 / 2}|\langle f, \varphi\rangle| \chi_{Q}(x)\right)^{q} d x\right\}^{1 / q}
$$

for $1 \leq q<\infty$, and

$$
\|f\|_{C M O_{p}^{\alpha, \infty}}:=\sup _{Q \in \mathscr{Q}}|Q|^{-\alpha / n-1 / p+1 / 2}\left|\left\langle f, \varphi_{Q}\right\rangle\right|
$$

Here $\chi_{Q}$ denotes the characteristic function of $Q$ and $q^{\prime}$ is the conjugate index of $q$, i.e., $1 / q+1 / q^{\prime}=1$. Throughout the article, $q^{\prime}$ is defined as $q^{\prime}=\infty$ whenever $0<q \leq 1$.

They introduced a new kind of sequence space $c_{p}^{\alpha, q}$, and then characterized the duals of $\dot{f}_{p}^{\alpha, q}$ by means of $c_{p}^{\alpha, q}$. Let us recall the definitions of the sequence spaces $\dot{f}_{p}^{\alpha, q}$ defined in 6 .

For $\alpha \in \mathbb{R}$ and $0<p, q \leq \infty$, the space $\dot{f}_{p}^{\alpha, q}$ consists of all such sequences $s=\left\{s_{Q}\right\}_{Q \in \mathscr{Q}}$ satisfying $\|s\|_{\dot{f}_{p}^{\alpha, q}}<\infty$, where

$$
\|s\|_{f_{p}^{\alpha, q}}:=\left\|\left\{\sum_{Q \in \mathscr{Q}}\left(|Q|^{-\alpha / n-1 / 2}\left|s_{Q}\right| \chi_{Q}\right)^{q}\right\}^{1 / q}\right\|_{L^{p}}
$$

if $0<p<\infty$ and

$$
\|s\|_{f_{\infty}^{\alpha, q}}:=\sup _{P \in \mathscr{Q}}\left\{|P|^{-1} \int_{P} \sum_{Q \in \mathscr{Q}_{P}}\left(|Q|^{-\alpha / n-1 / 2}\left|s_{Q}\right| \chi_{Q}(x)\right)^{q} d x\right\}^{1 / q}
$$


As before, the above $\ell^{q}$-norm is modified to the supremum norm for $0<p<\infty$ and $q=\infty$. For $p=q=\infty$, we adopt the norm

$$
\|\boldsymbol{s}\|_{\dot{f}_{\infty}^{\alpha, \infty}}:=\sup _{Q \in \mathscr{Q}}|Q|^{-\alpha / n-1 / 2}\left|s_{Q}\right|
$$

To study the dual of $\dot{f}_{p}^{\alpha, q}$, they introduced a discrete version of Carleson measure spaces $c_{p}^{\alpha, q}$. For $\alpha \in \mathbb{R}$ and $0<p \leq 1 \leq q \leq \infty$, the space $c_{p}^{\alpha, q}$ is the collection of all sequences $\boldsymbol{t}=\left\{t_{Q}\right\}_{Q \in \mathscr{Q}}$ satisfying $\|\boldsymbol{t}\|_{c_{p}^{\alpha, q}}<\infty$, where

$$
\|\boldsymbol{t}\|_{c_{p}^{\alpha, q}}:=\sup _{P \in \mathscr{Q}}\left\{|P|^{-q\left(1 / p-1 / q^{\prime}\right)} \int_{P} \sum_{Q \in \mathscr{Q}_{P}}\left[|Q|^{-\alpha / n-1 / 2}\left|t_{Q}\right| \chi_{Q}(x)\right]^{q} d x\right\}^{1 / q}
$$

for $1 \leq q<\infty$, and

$$
\|\boldsymbol{t}\|_{c_{p}^{\alpha, \infty}}:=\sup _{Q \in \mathscr{Q}}|Q|^{-\alpha / n-1 / p+1 / 2}\left|t_{Q}\right| .
$$

For $1 \leq q<\infty$ and $f \in C M O_{p}^{\alpha, q}$, let $S_{\varphi}(f):=\left\{\left\langle f, \varphi_{Q}\right\rangle\right\}_{Q}=\left\{s_{Q}\right\}_{Q}=s$. Then the $\varphi$-tranform identity shows $f=\sum_{Q} s_{Q} \psi_{Q}$ and $\|f\|_{C M O_{p}^{\alpha, q}}=\left\|S_{\varphi}(f)\right\|_{c_{p}^{\alpha, q}}=\|s\|_{c_{p}^{\alpha, q}}$. In particular, $\|f\|_{C M O_{1}^{\alpha, q}}=\left\|S_{\varphi}(f)\right\|_{c_{1}^{\alpha, q}}=\left\|S_{\varphi}(f)\right\|_{\dot{f}_{\infty}^{\alpha, q}} \approx\|f\|_{\dot{F}_{\infty}^{\alpha, q}}$. Furthermore, for $s \in c_{p}^{\alpha, q}$,

$$
\left\|T_{\psi}(\boldsymbol{s})\right\|_{C M O_{p}^{\alpha, q}}=\left\|\sum_{P} s_{P} \psi_{P}\right\|_{C M O_{p}^{\alpha, q}}=\left\|\left\{\left\langle\sum_{P} s_{P} \psi_{P}, \varphi_{Q}\right\rangle\right\}_{Q}\right\|_{c_{p}^{\alpha, q}}=\|A s\|_{c_{p}^{\alpha, q}},
$$

where $T_{\psi}(s):=\sum_{Q} s_{Q} \psi_{Q}$ and $A:=\left\{\left\langle\psi_{P}, \varphi_{Q}\right\rangle\right\}_{Q, P}$ is $\left(\alpha+n q\left(1 / p-1 / q^{\prime}\right), p, q\right)$-almost diagonal (cf. [6, Lemma 3.6]). They summarized that $\left.T_{\psi} \circ S_{\varphi}\right|_{C M O_{p}^{\alpha, q}}$ is also the identity on $C M O_{p}^{\alpha, q}$.

In 3 5], Bui and Taibleson defined another weighted version of Triebel-Linzorkin spaces. There are some other papers concerning this topic, see [2,7,8] for more details.

Given a weight $w$, let $\mathscr{Q}(w)$ denote the collection of all dyadic cubes $Q \subseteq \mathbb{R}^{n}$ such that $w(Q):=\int_{Q} w(Q) d x \neq 0$ and for $k \in \mathbb{Z}, \mathscr{Q}_{k}(w)$ denote the subcollections of $\mathscr{Q}(w)$ with side length $2^{-k}$. Also, for $P \in \mathscr{Q}(w), \mathscr{Q}_{P}(w)$ denotes the collection of all dyadic cubes $Q \in$ $\mathscr{Q}(w)$ with $Q \subseteq P$ and $\mathscr{Q}_{P, k}(w)$ denotes the collection of all dyadic cubes satisfying $Q \subseteq P$ and $\ell(Q)=2^{-k}$. Note that $\mathscr{Q}(w)=\bigcup_{k \in \mathbb{Z}} \mathscr{Q}_{k}(w)$, and $\mathscr{Q}_{P}(w)=\bigcup_{k \geq-\log _{2} \ell(P)} \mathscr{Q}_{P, k}(w)$.

In weighted cases, we adopt similar definitions for $S_{\varphi}$ and $T_{\psi}$ as follows. Define a linear map $S_{\varphi}$ from $\mathscr{S}^{\prime} / \mathscr{P}$ into the family of complex sequences by

$$
S_{\varphi}(f)=\left\{\left\langle f, \varphi_{Q}\right\rangle\right\}_{Q \in \mathscr{Q}(w)},
$$

and another linear map $T_{\psi}$ from the family of complex sequences into $\mathscr{S}^{\prime} / \mathscr{P}$ by

$$
T_{\psi}\left(\left\{s_{Q}\right\}_{Q \in \mathscr{Q}(w)}\right)=\sum_{Q \in \mathscr{Q}(w)} s_{Q} \psi_{Q} .
$$


In this article, we study the weighted generalized Carleson measure spaces via the $\varphi$-transform identity. To do so, we first need the following definitions.

Definition 1.1. For $\alpha \in \mathbb{R}, 0<p, q \leq+\infty$, and a weight $w$, we say that $f$ belongs to the homogeneous weighted Triebel-Lizorkin space $\dot{F}_{p, w}^{\alpha, q}$ if $f \in \mathscr{S}^{\prime} / \mathscr{P}$ satisfies $\|f\|_{\dot{F}_{p, w}^{\alpha, q}}<\infty$, where

$$
\|f\|_{\dot{F}_{p, w}^{\alpha, q}}:= \begin{cases}\left\|\left\{\sum_{k \in \mathbb{Z}}\left(2^{k \alpha}\left|\varphi_{k} * f\right|\right)^{q}\right\}^{1 / q}\right\|_{L^{p}(w)} & \text { for } p<\infty, \\ \sup _{P \in \mathscr{Q}(w)}\left\{|P|^{-1} \int_{P} \sum_{k=-\log _{2} \ell(P)}^{\infty}\left(2^{k \alpha}\left|\varphi_{k} * f(x)\right|\right)^{q} w(x) d x\right\}^{1 / q} & \text { for } p=\infty .\end{cases}
$$

Definition 1.2. Let $\varphi \in \mathscr{S}$ satisfy the conditions in (1.1). For $\alpha \in \mathbb{R}, 0<p \leq 1$, $0<q \leq \infty$ and a weight $w$, the weighted generalized Carleson measure space $\dot{C} M O_{p, w}^{\alpha, q}$ is the collection of all $f \in \mathscr{S}^{\prime} / \mathscr{P}$ satisfying $\|f\|_{\dot{C} M O_{p, w}^{\alpha, q}}<\infty$, where

$$
\begin{aligned}
& \|f\|_{\dot{C} M O_{p, w}^{\alpha, q}} \\
:= & \sup _{P \in \mathscr{Q}(w)}\left\{|P|^{-q\left(1 / p-1 / q^{\prime}\right)} \int_{P} \sum_{Q \in \mathscr{Q}_{P}(w)}\left(|Q|^{-\alpha / n-1 / 2}\left|\left\langle f, \varphi_{Q}\right\rangle\right| \chi_{Q}(x)\right)^{q} w(x) d x\right\}^{1 / q}
\end{aligned}
$$

for $0<q<\infty$, and

$$
\|f\|_{\dot{C} M O_{P, w}^{\alpha, \infty}}:=\sup _{P \in \mathscr{Q}(w)}|P|^{1-1 / p} \sup _{Q \in \mathscr{Q}_{P}(w)}|Q|^{-\alpha / n-1 / 2}\left|\left\langle f, \varphi_{Q}\right\rangle\right| .
$$

In order to prove that the definition of $\dot{C} M O_{p, w}^{\alpha, q}$ is independent of the choice of $\varphi \in \mathscr{S}$ satisfying certain conditions, we need the following Plancherel-Pôlya inequality (for $q=\infty$ and the other case we will give descriptions and prove them in Section 3).

Theorem 1.3 (Plancherel-Pôlya inequality). Let $\varphi, \phi \in \mathscr{S}$ satisfy $(1.1)$. For $\alpha \in \mathbb{R}$, $0<p \leq 1<q<\infty$ and a weight $w$ with doubling exponent $\beta$, if $f \in \mathscr{S}^{\prime} / \mathscr{P}$ satisfies

$$
\sup _{P \in \mathscr{Q}(w)}\left\{|P|^{-q\left(1 / p-1 / q^{\prime}\right)} \sum_{k=-\log _{2} \ell(P)}^{\infty} \sum_{Q \in \mathscr{Q}_{P, k}(w)}\left(2^{k \alpha} \sup _{u \in Q}\left|\widetilde{\varphi}_{k} * f(u)\right|\right)^{q} w(Q)\right\}^{1 / q}<\infty
$$

then

$$
\begin{aligned}
& \sup _{P \in \mathscr{Q}(w)}\left\{|P|^{-q\left(1 / p-1 / q^{\prime}\right)} \sum_{k=-\log _{2} \ell(P)}^{\infty} \sum_{Q \in \mathscr{Q}_{P, k}(w)}\left(2^{k \alpha} \sup _{u \in Q}\left|\widetilde{\phi}_{k} * f(u)\right|\right)^{q} w(Q)\right\}^{1 / q} \\
\approx & \sup _{P \in \mathscr{Q}(w)}\left\{|P|^{-q\left(1 / p-1 / q^{\prime}\right)} \sum_{k=-\log _{2} \ell(P)}^{\infty} \sum_{Q \in \mathscr{Q}_{P, k}(w)}\left(2^{k \alpha} \inf _{u \in Q}\left|\widetilde{\varphi}_{k} * f(u)\right|\right)^{q} w(Q)\right\}^{1 / q} .
\end{aligned}
$$


By the theorem above we can make the following remark.

Remark 1.4. Let $\varphi, \phi \in \mathscr{S}$ satisfy (1.1). For $\alpha \in \mathbb{R}, 0<p \leq 1<q<\infty$ and a weight $w$ with doubling exponent $\beta$. Denote $\dot{C} M O_{p, w}^{\alpha, q}(\varphi)$ as the collection of all $f \in \mathscr{S}^{\prime} / \mathscr{P}$ satisfying $\|f\|_{\dot{C} M O_{p, w}^{\alpha, q}(\varphi)}<\infty$ defined in Definition 1.2 with respect to $\varphi$. Then, by Theorem 1.3 .

$$
\begin{aligned}
& \|f\|_{\dot{C} M O_{p, w}^{\alpha, q}(\phi)} \\
\leq & \sup _{P \in \mathscr{Q}(w)}\left\{|P|^{-q\left(1 / p-1 / q^{\prime}\right)} \sum_{k=-\log _{2} \ell(P)}^{\infty} \sum_{Q \in \mathscr{Q}_{P, k}(w)}\left(2^{k \alpha} \sup _{u \in Q}\left|\widetilde{\phi}_{k} * f(u)\right|\right)^{q} w(Q)\right\}^{1 / q} \\
\leq & C \sup _{P \in \mathscr{Q}(w)}\left\{|P|^{-q\left(1 / p-1 / q^{\prime}\right)} \sum_{k=-\log _{2} \ell(P)}^{\infty} \sum_{Q \in \mathscr{Q}_{P, k}(w)}\left(2^{k \alpha} \inf _{u \in Q}\left|\widetilde{\varphi}_{k} * f(u)\right|\right)^{q} w(Q)\right\}^{1 / q} \\
\leq & C\|f\|_{\dot{C} M O_{P, w}^{\alpha, q}(\varphi)} .
\end{aligned}
$$

Similarly, $\|f\|_{\dot{C} M O_{p, w}^{\alpha, q}(\varphi)} \leq C\|f\|_{\dot{C} M O_{p, w}^{\alpha, q}(\phi)}$ by interchanging the roles of $\varphi$ and $\phi$. Hence the definition of $\dot{C} M O_{p, w}^{\alpha, q}(\varphi)$ is independent of the choice of $\varphi$ and, in short form, is denoted by $\dot{C} M O_{p, w}^{\alpha, q}$.

In order to obtain a norm equivalence, we need to define a discrete version of weighted Carleson measure spaces $\dot{c}_{p, w}^{\alpha, q}$. Before giving the definition of these spaces, let us recall the weighted homogeneous Triebel-Lizorkin sequence spaces.

Definition 1.5. For $\alpha \in \mathbb{R}, 0<p, q \leq \infty$ and a weight $w$, the space $\dot{f}_{p, w}^{\alpha, q}$ consists of all such sequences $s=\left\{s_{Q}\right\}_{Q \in \mathscr{Q}(w)}$ satisfying $\|s\|_{\dot{f}_{p, w}^{\alpha, q}}<\infty$, where

$$
\|\boldsymbol{s}\|_{\dot{f}_{p, w}^{\alpha, q}}:=\left\|\left\{\sum_{Q \in \mathscr{Q}}\left(|Q|^{-\alpha / n-1 / 2}\left|s_{Q}\right| \chi_{Q}\right)^{q}\right\}^{1 / q}\right\|_{L^{p}(w)}
$$

if $0<p<\infty$ and

$$
\|s\|_{f_{\infty, w}^{\alpha, q}}:=\sup _{P \in \mathscr{Q}(w)}\left\{|P|^{-1} \int_{P} \sum_{Q \in \mathscr{Q}_{P}(w)}\left(|Q|^{-\alpha / n-1 / 2}\left|s_{Q}\right| \chi_{Q}(x)\right)^{q} w(x) d x\right\}^{1 / q} .
$$

As before, the above $\ell^{q}$-norm is modified to the supremum norm for $0<p<\infty$ and $q=\infty$. For $p=q=\infty$, we adopt the norm

$$
\|s\|_{f_{\infty, w}^{\alpha, \infty}}:=\sup _{Q \in \mathscr{Q}(w)}|Q|^{-\alpha / n-1 / 2}\left|s_{Q}\right| .
$$

Next, let us define the weighted generalized Carleson measure sequence spaces. 
Definition 1.6. Let $w$ be a weight. For $\alpha \in \mathbb{R}, 0<p \leq 1$ and $0<q \leq \infty$, the space $\dot{c}_{p, w}^{\alpha, q}$ is the collection of all sequences $\boldsymbol{t}=\left\{t_{Q}\right\}_{Q \in \mathscr{Q}(w)}$ satisfying $\|\boldsymbol{t}\|_{\dot{c}_{p, w}^{\alpha, q}}<\infty$, where

$$
\|\boldsymbol{t}\|_{\dot{c}_{p, w}^{\alpha, q}}:=\sup _{P \in \mathscr{Q}(w)}\left\{|P|^{-q\left(1 / p-1 / q^{\prime}\right)} \int_{P} \sum_{Q \in \mathscr{Q}_{P}(w)}\left[|Q|^{-\alpha / n-1 / 2}\left|t_{Q}\right| \chi_{Q}(x)\right]^{q} w(x) d x\right\}^{1 / q}
$$

for $0<q<\infty$, and

$$
\|\boldsymbol{t}\|_{\dot{c}_{p, w}^{\alpha, \infty}}:=\sup _{P \in \mathscr{Q}(w)}|P|^{1-1 / p} \sup _{Q \in \mathscr{Q}_{P}(w)}|Q|^{-\alpha / n-1 / 2}\left|t_{Q}\right| .
$$

As a consequence of Plancherel-Pôlya inequalities, we have a result concerning the norm equivalence between generalized function spaces and corresponding sequence spaces.

Theorem 1.7. Suppose $\alpha \in \mathbb{R}, 0<p \leq 1,0<q \leq \infty, w \in A_{\infty}$ and $\varphi, \psi$ in $\mathscr{S}$ satisfy (1.1) and 1.2. The linear operators $S_{\varphi}: \dot{C} M O_{p, w}^{\alpha, q} \mapsto \dot{c}_{p, w}^{\alpha, q}$ and $T_{\psi}: \dot{c}_{p, w}^{\alpha, q} \mapsto \dot{C} M O_{p, w}^{\alpha, q}$ defined by (1.4) and (1.5), respectively, are bounded. Furthermore $T_{\psi} \circ S_{\varphi}$ is the identity on $\dot{C} M O_{p, w}^{\alpha, q}$. In particular, $\|f\|_{\dot{C} M O_{1, w}^{\alpha, q}}=\left\|S_{\varphi}(f)\right\|_{\dot{c}_{1, w}^{\alpha, q}}=\left\|S_{\varphi}(f)\right\|_{\dot{f}_{\infty, w}^{\alpha, q}} \approx\|f\|_{\dot{F}_{\infty, w}^{\alpha, q}}$.

The organization of this article is as follows. We recall weights and some preliminary results in Section 2, In Section 3, we show the Plancherel-Pôlya inequalities that give us the independence of the choice of $\varphi$ for the definition of weighted generalized Carleson measure spaces. In Section 4, we show a norm equivalence between $\dot{C} M O_{p, w}^{\alpha, q}$ and $\dot{c}_{p, w}^{\alpha, q}$. Through the article, we use $j \wedge k$ to denote the minimum of $j$ and $k$, use $j \vee k$ to denote the maximum of $j$ and $k$, and use $C$ to denote a positive constant independent of the main variables, which may vary from line to line.

\section{Weights}

We say that $w$ is a weight if $w$ is a non-negative measurable function on $\mathbb{R}^{n}$. At the beginning of this section, let us recall the definition of "doubling condition".

Definition 2.1. A weight $w$ is called a doubling measure, if there exists a constant $C=C_{n}$ such that for any $\delta>0$ and any $z \in \mathbb{R}^{n}$,

$$
\int_{B_{2 \delta}(z)} w(t) d t \leq C \int_{B_{\delta}(z)} w(t) d t
$$

where $B_{\delta}(z)$ is an open ball in $\mathbb{R}^{n}$ centered at $z$ with radius $\delta$. If $C=2^{\beta}$ is the smallest constant such that the inequality (2.1) holds, then $\beta$ is called the doubling exponent of $w$.

Here, we recall the definition of $A_{p}$ weights. For $s>0$ and a weight $w$, define $w^{-s}$ by

$$
w^{-s}(x)= \begin{cases}{[w(x)]^{-s}} & \text { if } w(x) \neq 0 \\ 0 & \text { otherwise }\end{cases}
$$


Definition 2.2 $\left(A_{p}\right.$ weights). Let $w$ be a non-negative and locally integrable function on $\mathbb{R}^{n}$. We say that $w \in A_{p}$ if $\|w\|_{A_{p}}$ is finite, where $\|w\|_{A_{p}}$ is defined by

$$
\|w\|_{A_{p}}= \begin{cases}\sup _{Q} \operatorname{ess}_{\sup _{y \in Q} w^{-1}(y) \frac{1}{|Q|} \int_{Q} w(t) d t} & \text { if } p=1 \\ \sup _{Q}\left(\frac{1}{|Q|} \int_{Q} w(x) d x\right)\left(\frac{1}{|Q|} \int_{Q} w^{1-p^{\prime}}(x) d x\right)^{p-1} & \text { if } 1<p<\infty\end{cases}
$$

where the suprema are taken over all cubes (with sides parallel to the coordinate axes) in $\mathbb{R}^{n}$. We also set $A_{\infty}=\bigcup_{1 \leq p<\infty} A_{p}$.

Note that we can replace any cubes by any balls in $\mathbb{R}^{n}$ in the last definition. Also note that when $w \in A_{p}$ for $1 \leq p \leq \infty, w(x) d x$ is a doubling measure (see [13, Theorem 2.1, p. 226]).

The following is a characterization of Muckenhoupt $A_{p}$ weights.

Lemma 2.3. Let $1<q<\infty$ and $w \in A_{q}$. Then, for every cube $Q \subseteq \mathbb{R}^{n}$

$$
|Q| \leq[w(Q)]^{1 / q}\left[w^{1-q^{\prime}}(Q)\right]^{(q-1) / q} \leq C|Q|,
$$

where $C$ is dependent only on the constant of $A_{q}$ condition. Moreover,

$$
\left(\frac{|Q|}{w(Q)}\right)^{q^{\prime}} w(Q) \approx w^{1-q^{\prime}}(Q)
$$

Proof. By Hölder's inequality and $A_{q}$ condition, we have, for a cube $Q$ in $\mathbb{R}^{n}$

$$
\begin{aligned}
|Q| & \leq\left(\int_{Q} w(x) d x\right)^{1 / q}\left(\int_{Q} w^{-q^{\prime} / q}(x) d x\right)^{-1 / q^{\prime}} \\
& =\left\{[w(Q)]\left[w^{-\left(q^{\prime}-1\right)}(Q)\right]^{q-1}\right\}^{1 / q} \leq C|Q| .
\end{aligned}
$$

That implies

$$
|Q|^{q} \leq w(Q)\left[w^{1-q^{\prime}}(Q)\right]^{q-1} \leq C|Q|^{q}
$$

then

$$
1 \leq \frac{w(Q)}{|Q|}\left(\frac{w^{1-q^{\prime}}(Q)}{|Q|}\right)^{q-1} \leq C
$$

Therefore, we have

$$
\begin{aligned}
\left(\frac{|Q|}{w(Q)}\right)^{q^{\prime}} w(Q) & =\left(\frac{|Q|}{w(Q)}\right)^{q^{\prime}-1}|Q| \\
& \approx\left(\frac{w^{1-q^{\prime}}(Q)}{|Q|}\right)^{(q-1)\left(q^{\prime}-1\right)}|Q|=w^{1-q^{\prime}}(Q),
\end{aligned}
$$

and the proof is finished. 
Also there is a weighted version of Fefferman-Stein vector-valued maximal inequality for Hardy-Littlewood maximal function $M$, which is given below.

Proposition 2.4. [1] Let $1<p, q<\infty$ and $w \in A_{p}$. There is a constant $C=C_{p}$ independent of $\left\{f_{i}\right\}_{i}$ such that

$$
\left\|\left(\sum_{i}\left|M f_{i}\right|^{q}\right)^{1 / q}\right\|_{L^{p}(w)} \leq C\left\|\left(\sum_{i}\left|f_{i}\right|^{q}\right)^{1 / q}\right\|_{L^{p}(w)}
$$

for any $\left\{f_{i}\right\}_{i} \in L^{p}(w)\left(\ell^{q}\right)$.

\section{Plancherel-Pôlya inequalities}

In this section, we show Plancherel-Pôlya inequalities that give us the independence of the choice of $\varphi$ for the definition of weighted Carleson measure spaces. Before proving those, let us recall a basic estimate of Roudenko 12 .

Lemma 3.1. [12 Let $w$ be a weight with doubling exponent $\beta$. If $L>\beta$, then for $r \geq \ell(Q)$,

$$
\int_{\mathbb{R}^{n}} w(x)\left(1+\frac{\left|x-x_{Q}\right|}{r}\right)^{-L} d x \leq c_{\beta}\left[\frac{r}{\ell(Q)}\right]^{\beta} \int_{Q} w(x) d x .
$$

Now we can prove the following Plancherel-Pôlya inequalities.

Proof of Theorem 1.3. Without loss of generality, we may assume $\alpha=0$. By the $\varphi$ transform identity, 1.3 , we rewrite $\widetilde{\phi}_{j} * f(u)$ as

$$
\begin{aligned}
\widetilde{\phi}_{j} * f(u) & =\sum_{Q \in \mathscr{Q}}\left\langle f, \varphi_{Q}\right\rangle \int \widetilde{\phi}_{j}(u-x) \psi_{Q}(x) d x \\
& =\sum_{k \in \mathbb{Z}} \sum_{Q \in \mathscr{Q}_{k}}|Q|\left\langle f, \varphi_{k}\left(\cdot-x_{Q}\right)\right\rangle \int \widetilde{\phi}_{j}(u-x) \psi_{k}\left(x-x_{Q}\right) d x
\end{aligned}
$$

Using the inequality (B.5) in 6, p. 151],

$$
\left|\int \widetilde{\phi}_{j}(u-x) \psi_{k}\left(x-x_{Q}\right) d x\right| \leq C 2^{-K|j-k|} \frac{2^{-(j \wedge k)(J-n)}}{\left(2^{-(j \wedge k)}+\left|u-x_{Q}\right|\right)^{J}},
$$

where $j \wedge k=\min \{j, k\}, J>\beta+n$ and $K>(\beta-n) \vee(J-\beta-n)$, we obtain

$$
\left|\widetilde{\phi}_{j} * f(u)\right| \leq C \sum_{k \in \mathbb{Z}} \sum_{Q \in \mathscr{Q}_{k}} 2^{-K|j-k|}|Q| \frac{2^{-(j \wedge k)(J-n)}}{\left(2^{-(j \wedge k)}+\left|u-x_{Q}\right|\right)^{J}}\left|\widetilde{\varphi}_{j} * f\left(x_{Q}\right)\right| .
$$


Thus, for $\ell\left(Q^{\prime}\right)=2^{-j}$,

$$
\begin{aligned}
\left(\sup _{u \in Q^{\prime}}\left|\widetilde{\phi}_{j} * f(u)\right|\right)^{q} & \leq C\left(\sum_{k \in \mathbb{Z}} \sum_{Q \in \mathscr{Q}_{k}} 2^{-K|j-k|}|Q| \frac{2^{-(j \wedge k)(J-n)}}{\left(2^{-(j \wedge k)}+\left|x_{Q^{\prime}}-x_{Q}\right|\right)^{J}}\left|\widetilde{\varphi}_{k} * f\left(x_{Q}\right)\right|\right)^{q} \\
& \leq C \sum_{k \in \mathbb{Z}} \sum_{Q \in \mathscr{Q}_{k}} 2^{-K|j-k|}|Q| \frac{2^{-(j \wedge k)(J-n)}}{\left(2^{-(j \wedge k)}+\left|x_{Q^{\prime}}-x_{Q}\right|\right)^{J}}\left|\widetilde{\varphi}_{k} * f\left(x_{Q}\right)\right|^{q},
\end{aligned}
$$

where the last inequality is followed by Hölder's inequality and

$$
\sum_{Q \in \mathscr{Q}_{k}}|Q| \frac{2^{-(j \wedge k)(J-n)}}{\left(2^{-(j \wedge k)}+\left|x_{Q^{\prime}}-x_{Q}\right|\right)^{J}} \leq C .
$$

Denote $T_{Q}$ by

$$
T_{Q}:=\inf _{u \in Q}\left|\widetilde{\varphi}_{k} * f(u)\right|^{q} .
$$

Since $x_{Q}$ can be replaced by any point in $Q$ in the last inequality,

$$
\left(\sup _{u \in Q^{\prime}}\left|\widetilde{\phi}_{j} * f(u)\right|\right)^{q} \leq C \sum_{k \in \mathbb{Z}} \sum_{Q \in \mathscr{Q}_{k}} 2^{-K|j-k|}|Q| \frac{2^{-(j \wedge k)(J-n)}}{\left(2^{-(j \wedge k)}+\left|x_{Q^{\prime}}-x_{Q}\right|\right)^{J}} T_{Q} .
$$

Given a dyadic cube $P$ with $\ell(P)=2^{-k_{0}}$, the above estimates yield

$$
\begin{aligned}
& \sum_{j=k_{0}}^{\infty} \sum_{Q^{\prime} \in \mathscr{Q}_{P, j}}\left(\sup _{u \in Q^{\prime}}\left|\widetilde{\phi}_{j} * f(u)\right|\right)^{q} w\left(Q^{\prime}\right) \\
\leq & C \sum_{j=k_{0}}^{\infty} \sum_{Q^{\prime} \in \mathscr{Q}_{P, j}} \sum_{k \in \mathbb{Z}} \sum_{Q \in \mathscr{Q}_{k}} 2^{-K|j-k|} \frac{2^{-(j \wedge k)(J-n)}}{\left(2^{-(j \wedge k)}+\left|x_{Q^{\prime}}-x_{Q}\right|\right)^{J}}|Q| \frac{w\left(Q^{\prime}\right)}{w(Q)} T_{Q} w(Q) \\
:= & C E_{1}+C E_{2},
\end{aligned}
$$

where

$$
E_{1}=\sum_{j=k_{0}}^{\infty} \sum_{Q^{\prime} \in \mathscr{Q}_{P, j}} \sum_{k \geq j} \sum_{Q \in \mathscr{Q}_{k}} 2^{-K|j-k|} \frac{2^{-(j \wedge k)(J-n)}}{\left(2^{-(j \wedge k)}+\left|x_{Q^{\prime}}-x_{Q}\right|\right)^{J}}|Q| \frac{w\left(Q^{\prime}\right)}{w(Q)} T_{Q} w(Q)
$$

and

$$
E_{2}=\sum_{j=k_{0}}^{\infty} \sum_{Q^{\prime} \in \mathscr{Q}_{P, j}} \sum_{k<j} \sum_{Q \in \mathscr{Q}_{k}} 2^{-K|j-k|} \frac{2^{-(j \wedge k)(J-n)}}{\left(2^{-(j \wedge k)}+\left|x_{Q^{\prime}}-x_{Q}\right|\right)^{J}}|Q| \frac{w\left(Q^{\prime}\right)}{w(Q)} T_{Q} w(Q) .
$$

Then $E_{1}$ can be further decomposed as

$$
\begin{aligned}
& E_{1}=\sum_{j=k_{0}}^{\infty} \sum_{Q^{\prime} \in \mathscr{Q}_{P, j}} \sum_{k \geq j} \sum_{Q \in \mathscr{Q}_{3 P, k}} 2^{-K|j-k|} \frac{2^{-(j \wedge k)(J-n)}}{\left(2^{-(j \wedge k)}+\left|x_{Q^{\prime}}-x_{Q}\right|\right)^{J}}|Q| \frac{w\left(Q^{\prime}\right)}{w(Q)} T_{Q} w(Q) \\
& +\sum_{j=k_{0}}^{\infty} \sum_{Q^{\prime} \in \mathscr{Q}_{P, j}} \sum_{k \geq j} \sum_{\substack{Q \cap 3 P=\emptyset \\
Q \in \mathscr{Q}_{k}}} 2^{-K|j-k|} \frac{2^{-(j \wedge k)(J-n)}}{\left(2^{-(j \wedge k)}+\left|x_{Q^{\prime}}-x_{Q}\right|\right)^{J}}|Q| \frac{w\left(Q^{\prime}\right)}{w(Q)} T_{Q} w(Q) \\
& :=E_{11}+E_{12} \text {. }
\end{aligned}
$$


There are $3^{n}$ dyadic cubes in $3 P$ with the same side length as $P$, so if $P^{\prime} \subseteq 3 P$ then $\left|P^{\prime}\right|=|P|$ and

$$
\sum_{Q \in \mathscr{Q}_{3 P, k}} T_{Q} w(Q) \leq 3^{n} \sup _{\substack{P^{\prime} \subseteq 3 P \\ \ell\left(P^{\prime}\right)=\ell(P)}} \sum_{Q \in \mathscr{Q}_{P^{\prime}, k}} T_{Q} w(Q) .
$$

Let $J>\beta, k \geq j$ and $w$ be a weight with doubling exponent $\beta$. By Lemma 3.1, we have

$$
\int_{\mathbb{R}^{n}}\left(1+\frac{\left|x-x_{Q}\right|}{2^{-j}}\right)^{-J} w(x) d x \leq c_{\beta} 2^{(k-j) \beta} w(Q),
$$

and so

$$
\begin{aligned}
& \sum_{j=k_{0}}^{\infty} \sum_{Q^{\prime} \in \mathscr{Q}_{P, j}} 2^{-K|j-k|} \frac{2^{-(j \wedge k)(J-n)}}{\left(2^{-(j \wedge k)}+\left|x_{Q^{\prime}}-x_{Q}\right|\right)^{J}}|Q| w\left(Q^{\prime}\right) \\
\leq & C \sum_{j=k_{0}}^{\infty} 2^{-K(k-j)} 2^{j n} 2^{-k n} \int_{\mathbb{R}^{n}}\left(1+\frac{\left|x-x_{Q}\right|}{2^{-j}}\right)^{-J} w(x) d x \\
\leq & C w(Q) .
\end{aligned}
$$

Hence

$$
\begin{aligned}
|P|^{-q\left(1 / p-1 / q^{\prime}\right)} E_{11} \leq C|P|^{-q\left(1 / p-1 / q^{\prime}\right)} \sum_{j=k_{0}}^{\infty} \sum_{Q^{\prime} \in \mathscr{Q}_{P, j}} \sum_{k \geq j} \sum_{Q \in \mathscr{Q}_{3 P, k}} 2^{-K|j-k|} \\
\quad \times\left(\frac{2^{-(j \wedge k)(J-n)}}{\left(2^{-(j \wedge k)}+\left|x_{Q^{\prime}}-x_{P}\right|\right)^{J}}|Q| \frac{w\left(Q^{\prime}\right)}{w(Q)} T_{Q} w(Q)\right) \\
\leq C \sup _{P^{\prime} \in \mathscr{Q}(w)}\left|P^{\prime}\right|^{-q\left(1 / p-1 / q^{\prime}\right)} \sum_{k=-\log _{2} \ell}^{\infty} \sum_{\left(P^{\prime}\right)} \inf _{u \in \mathscr{Q}_{P^{\prime}, k}}\left|\widetilde{\varphi}_{k} * f(u)\right|^{q} w(Q) .
\end{aligned}
$$

Next, we decompose the set of dyadic cubes $\{Q: Q \cap 3 P=\emptyset, \ell(Q)=\ell(P)\}$ into $\left\{B_{i}\right\}_{i \in \mathbb{N}}$ according to the distance between each $Q$ and $P$. Namely, for each $i \in \mathbb{N}$,

$$
B_{i}:=\left\{P^{\prime} \in \mathscr{Q}: P^{\prime} \cap 3 P=\emptyset, \ell(P)=\ell\left(P^{\prime}\right), 2^{i-k_{0}} \leq\left\|y_{P^{\prime}}-y_{P}\right\|<2^{i-k_{0}+1}\right\}
$$

where $y_{Q}$ denotes the center of $Q$. Then we obtain

$$
\begin{aligned}
|P|^{-q\left(1 / p-1 / q^{\prime}\right)} E_{12} \leq C \sum_{i=1}^{\infty} \sum_{P^{\prime} \in B_{i}} & \left|P^{\prime}\right|^{-q\left(1 / p-1 / q^{\prime}\right)} \sum_{j=k_{0}}^{\infty} \sum_{Q^{\prime} \in \mathscr{Q}_{P, j}} \sum_{k \geq j} \sum_{\substack{Q \in \mathscr{Q}_{P^{\prime}, k} \\
P^{\prime} \in B_{i}}} 2^{-K|j-k|} \\
& \times\left(\frac{2^{-j(J-n)}}{\left(2^{-j}+\left|x_{Q^{\prime}}-x_{P}\right|\right)^{J}}|Q| \frac{w\left(Q^{\prime}\right)}{w(Q)} T_{Q} w(Q)\right) .
\end{aligned}
$$


Since $w\left(Q^{\prime}\right) / w(Q) \leq C 2^{\beta\left(k-k_{0}+i\right)}$ and $\left\|x_{P^{\prime}}-x_{P}\right\| \approx 2^{i-k_{0}}$ for $P^{\prime} \in B_{i}$, the right-hand side of the inequality above is dominated by

$$
\begin{aligned}
C \sum_{i=1}^{\infty} \sum_{P^{\prime} \in B_{i}} \sum_{j=k_{0}}^{\infty} \sum_{Q^{\prime} \in \mathscr{Q}_{P, j}} \sum_{k \geq j} \sum_{\substack{Q \in \mathscr{Q}_{P^{\prime}, k} \\
P^{\prime} \in B_{i}}} 2^{\beta\left(k-k_{0}+i\right)} 2^{-K(k-j)} 2^{-\left(i-k_{0}\right) J} 2^{-j(J-n)}|Q| \\
\quad \times\left(\sup _{P^{\prime} \in \mathscr{Q}(w)}\left|P^{\prime}\right|^{-q\left(1 / p-1 / q^{\prime}\right)} \sum_{k \geq k_{0}} \sum_{Q \in \mathscr{Q}_{P^{\prime}, k}} T_{Q} w(Q)\right) .
\end{aligned}
$$

Because there are at most $2^{(i+2) n}$ dyadic cubes in $B_{i}, J>\beta+n, K+n>\beta$ and $|Q|=\left|Q^{\prime}\right| \frac{|Q|}{\left|Q^{\prime}\right|}$,

$$
\begin{aligned}
& |P|^{-q\left(1 / p-1 / q^{\prime}\right)} E_{12} \\
\leq & C\left\{\sup _{P^{\prime} \in \mathscr{Q}(w)}\left|P^{\prime}\right|^{-q\left(1 / p-1 / q^{\prime}\right)} \sum_{k \geq k_{0}} \sum_{Q \in \mathscr{Q}_{P^{\prime}, k}} T_{Q} w(Q)\right\} \\
& \times\left[\sum_{i=1}^{\infty} \sum_{j=k_{0}}^{\infty} \sum_{k \geq j} 2^{\beta\left(k-k_{0}+i\right)} 2^{-K(k-j)} 2^{-\left(i-k_{0}\right) J} 2^{-(J-n) j} 2^{-k_{0} n} 2^{(j-k) n} 2^{i n}\right] \\
\leq & C \sup _{P^{\prime} \in \mathscr{Q}(w)}\left|P^{\prime}\right|^{-q\left(1 / p-1 / q^{\prime}\right)} \sum_{k=-\log _{2} \ell\left(P^{\prime}\right)}^{\infty} \sum_{Q \in \mathscr{Q}_{P^{\prime}, k}} \inf _{u \in Q}\left|\widetilde{\varphi}_{k} * f(u)\right|^{q} w(Q) .
\end{aligned}
$$

To estimate $E_{2}$, for $i \in \mathbb{N}$ and $k<k_{0}$, set

$$
G_{i, k}:=\left\{Q: \ell(Q)=2^{-k} \text { and } x_{Q} \in 2^{i+1} P \backslash 2^{i} P\right\}
$$

Then $\left|x_{Q}-x_{P}\right| \approx 2^{i-k_{0}}$ for $Q \in G_{i, k}$ and

$$
\begin{gathered}
E_{2}=\sum_{j=k_{0}}^{\infty} \sum_{Q^{\prime} \in \mathscr{Q}_{P, j}} \sum_{k<j} \sum_{i=1}^{\infty} \sum_{Q \in G_{i, k}} \frac{2^{-K|j-k|}}{|Q|^{-q\left(1 / p-1 / q^{\prime}\right)}} \frac{2^{-k(J-n)}}{\left(2^{-k}+\left|x_{Q^{\prime}}-x_{Q}\right|\right)^{J}} \frac{w\left(Q^{\prime}\right)}{w(Q)}|Q| \\
\times|Q|^{-q\left(1 / p-1 / q^{\prime}\right)} T_{Q} w(Q) .
\end{gathered}
$$

Since $J>\beta+n$ and $K>J-n-\beta$, there are at most $2^{\left(i+k-k_{0}\right) n}$ dyadic cubes contained in $G_{i, k}$ and

$$
|Q|^{-q\left(1 / p-1 / q^{\prime}\right)} T_{Q} w(Q) \leq \sup _{P^{\prime} \in \mathscr{Q}(w)}\left|P^{\prime}\right|^{-q\left(1 / p-1 / q^{\prime}\right)} \sum_{m \geq-\log _{2} \ell\left(P^{\prime}\right)} \sum_{R \in \mathscr{Q}_{P^{\prime}, m}} T_{R} w(R)
$$




$$
\begin{aligned}
|Q|^{-q\left(1 / p-1 / q^{\prime}\right)} E_{2} \leq & C\left\{\sup _{P^{\prime} \in \mathscr{Q}(w)}\left|P^{\prime}\right|^{-q\left(1 / p-1 / q^{\prime}\right)} \sum_{m \geq-\log _{2} \ell\left(P^{\prime}\right)} \sum_{Q \in \mathscr{Q}_{P^{\prime}, m}} T_{Q} w(Q)\right\} \\
& \times\left[\sum_{j=k_{0}}^{\infty} \sum_{k<j} \sum_{i=1}^{\infty} 2^{-K(j-k)} 2^{-\left(i-k_{0}\right) J} 2^{-k(J-n)} 2^{\beta\left(k-k_{0}+i\right)} 2^{-k n} 2^{\left(i+k-k_{0}\right) n}\right] \\
\leq & C \sup _{P^{\prime} \in \mathscr{Q}(w)}\left|P^{\prime}\right|^{-q\left(1 / p-1 / q^{\prime}\right)} \sum_{m \geq-\log _{2} \ell\left(P^{\prime}\right)} \sum_{Q \in \mathscr{Q}_{P^{\prime}, m}} \inf _{u \in Q}\left|\widetilde{\varphi}_{m} * f(u)\right|^{q} w(Q) .
\end{aligned}
$$

According to the estimates of $E_{1}$ and $E_{2}$, we complete the proof.

For $q=\infty$ and $q \leq 1$ we have following results.

Theorem 3.2 (Plancherel-Pôlya inequality for $q=\infty$ ). Let $\varphi, \phi \in \mathscr{S}$ satisfy (1.1). For $\alpha \in \mathbb{R}, 0<p \leq 1, q=\infty$ and a weight $w$ with doubling exponent $\beta$, if $f \in \mathscr{S}^{\prime} / \mathscr{P}$ satisfies

$$
\sup _{P \in \mathscr{Q}(w)}|P|^{1-1 / p} \sup _{\substack{Q \in \mathscr{Q}_{P, j} \\ j \geq-\log _{2} \ell(P)}} 2^{j \alpha} \sup _{u \in Q}\left|\widetilde{\varphi}_{j} * f(u)\right|<\infty,
$$

then

$$
\begin{aligned}
& \sup _{P \in \mathscr{Q}(w)}|P|^{1-1 / p} \sup _{\substack{Q \in \mathscr{Q}_{P, j} \\
j \geq-\log _{2} \ell(P)}} 2^{j \alpha} \sup _{u \in Q}\left|\widetilde{\varphi}_{j} * f(u)\right| \\
\approx & \sup _{P \in \mathscr{Q}(w)}|P|^{1-1 / p} \sup _{\substack{Q \in \mathscr{Q}_{P, j} \\
j \geq-\log _{2} \ell(P)}} 2^{j \alpha} \inf _{u \in Q}\left|\widetilde{\phi}_{j} * f(u)\right| .
\end{aligned}
$$

Proof. Without loss of generality, we may assume $\alpha=0$. By a similar argument as the proof of Theorem 1.3 , we have

$$
\sup _{u \in Q^{\prime}}\left|\widetilde{\phi}_{j} * f(u)\right| \leq C \sum_{k \in \mathbb{Z}} \sum_{Q \in \mathscr{Q}_{k}} 2^{-K|j-k|}|Q| \frac{2^{-(j \wedge k)(J-n)}}{\left(2^{-(j \wedge k)}+\left|x_{Q^{\prime}}-x_{Q}\right|\right)^{J}} T_{Q},
$$

where $J>n, K>J-n$ and

$$
T_{Q}:=\inf _{u \in Q}\left|\widetilde{\varphi}_{k} * f(u)\right| .
$$

Given a dyadic cube $P$ with $\ell(P)=2^{-k_{0}}$, the above estimates yield

$$
\begin{aligned}
& \sup _{\substack{Q^{\prime} \in \mathscr{Q}_{P, j} \\
j \geq-\log _{2} \ell(P)}} \sup _{u \in Q^{\prime}}\left|\widetilde{\phi}_{j} * f(u)\right| \\
& \leq C \sup _{\substack{Q^{\prime} \in \mathscr{Q}_{P, j} \\
j \geq-\log _{2} \ell(P)}} \sum_{k \in \mathbb{Z}} \sum_{Q \in \mathscr{Q}_{k}} 2^{-K|j-k|}|Q| \frac{2^{-(j \wedge k)(J-n)}}{\left(2^{-(j \wedge k)}+\left|x_{Q^{\prime}}-x_{Q}\right|\right)^{J}} T_{Q} \\
&:=C E_{1}+C E_{2},
\end{aligned}
$$


where

$$
E_{1}=\sup _{\substack{Q^{\prime} \in \mathscr{Q}_{P, j} \\ j \geq-\log _{2} \ell(P)}} \sum_{k \geq j} \sum_{Q \in \mathscr{Q}_{k}} 2^{-K|j-k|}|Q| \frac{2^{-(j \wedge k)(J-n)}}{\left(2^{-(j \wedge k)}+\left|x_{Q^{\prime}}-x_{Q}\right|\right)^{J}} T_{Q}
$$

and

$$
E_{2}=\sup _{\substack{Q^{\prime} \in \mathscr{Q}_{P, j} \\ j \geq-\log _{2} \ell(P)}} \sum_{k<j} \sum_{Q \in \mathscr{Q}_{k}} 2^{-K|j-k|}|Q| \frac{2^{-(j \wedge k)(J-n)}}{\left(2^{-(j \wedge k)}+\left|x_{Q^{\prime}}-x_{Q}\right|\right)^{J}} T_{Q} .
$$

Then $E_{1}$ can be further decomposed as

$$
\begin{aligned}
E_{1}= & \sup _{\substack{Q^{\prime} \in \mathscr{Q}_{P, j} \\
j \geq-\log _{2} \ell(P)}} \sum_{k \geq j} \sum_{Q \in \mathscr{Q}_{3 P, k}} 2^{-K|j-k|}|Q| \frac{2^{-(j \wedge k)(J-n)}}{\left(2^{-(j \wedge k)}+\left|x_{Q^{\prime}}-x_{Q}\right|\right)^{J}} T_{Q} \\
& +\sup _{\substack{Q^{\prime} \in \mathscr{Q}_{P, j} \\
j \geq-\log _{2} \ell(P)}} \sum_{\substack { k \geq j \\
\begin{subarray}{c}{Q \cap 3 P=\emptyset \\
Q \in \mathscr{Q}_{k}{ k \geq j \\
\begin{subarray} { c } { Q \cap 3 P = \emptyset \\
Q \in \mathscr { Q } _ { k } } }\end{subarray}} 2^{-K|j-k|}|Q| \frac{2^{-(j \wedge k)(J-n)}}{\left(2^{-(j \wedge k)}+\left|x_{Q^{\prime}}-x_{Q}\right|\right)^{J}} T_{Q} \\
:= & E_{11}+E_{12} .
\end{aligned}
$$

There are $3^{n}$ dyadic cubes in $3 P$ with the same side length as $P$, so if $P^{\prime} \subseteq 3 P$ then $\left|P^{\prime}\right|=|P|$. Thus

$$
\begin{aligned}
& |P|^{1-1 / p} E_{11} \leq C|P|^{1-1 / p} \sup _{\substack{Q^{\prime} \in \mathscr{Q}_{P, j} \\
j \geq-\log _{2} \ell(P)}} \sum_{k \geq j} \sum_{\substack{P^{\prime} \in \mathscr{Q}_{3 P} \\
\ell\left(P^{\prime}\right)=\ell(P)}} 2^{-K|j-k|} \\
& \times\left(\sum_{Q \in \mathscr{Q}_{P^{\prime}, k}}|Q| \frac{2^{-j(J-n)}}{\left(2^{-j}+\left|x_{Q^{\prime}}-x_{P}\right|\right)^{J}} \sup _{\substack{Q \in \mathscr{Q}_{P^{\prime}, k} \\
k \geq-\log _{2} \ell\left(P^{\prime}\right)}} T_{Q}\right) \\
& \leq C|P|^{1-1 / p} \sum_{k \geq j} 2^{-K(k-j)} \sup _{\substack{Q \in \mathscr{Q}_{P^{\prime}, k} \\
k \geq-\log _{2} \ell\left(P^{\prime}\right)}} T_{Q} \\
& \leq C \sup _{P^{\prime} \in \mathcal{Q}(w)}\left|P^{\prime}\right|^{1-1 / p} \sup _{\substack{Q \in \mathscr{Q}_{P^{\prime}, k} \\
k \geq-\log _{2} \ell\left(P^{\prime}\right)}} \inf _{u \in Q}\left|\widetilde{\varphi}_{k} * f(u)\right|,
\end{aligned}
$$

since $\sum_{Q \in \mathscr{Q}_{k}}|Q| \frac{2^{-j(J-n)}}{\left(2^{-j}+\left|x_{Q^{\prime}}-x_{P}\right|\right)^{J}}$ is independent of $k \in \mathbb{N}$.

Next, we decompose the set of dyadic cubes $\{Q: Q \cap 3 P=\emptyset, \ell(Q)=\ell(P)\}$ into $\left\{B_{i}\right\}_{i \in \mathbb{N}}$ as 3.2 . Then we obtain

$$
\begin{aligned}
& |P|^{1-1 / p} E_{12}
\end{aligned}
$$

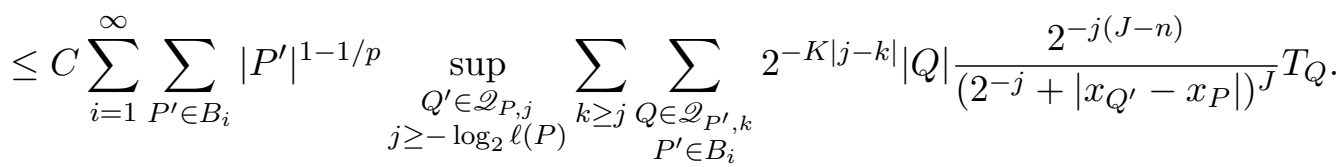


There are at most $2^{(i+2) n}$ dyadic cubes in $B_{i}$ with length $\ell(P)$. Since $\left|x_{P^{\prime}}-x_{P}\right| \approx 2^{i-k_{0}}$ for $P^{\prime} \in B_{i}$, the right-hand side of the inequality is dominated by

$$
C \sum_{i=1}^{\infty} \sum_{P^{\prime} \in B_{i}} \sum_{k \geq j} 2^{-K(k-j)} 2^{-k n} 2^{-\left(i-k_{0}\right) J} 2^{-j(J-n)} \times\left(\left|P^{\prime}\right|^{1-1 / p} \sup _{\substack{Q \in \mathscr{Q}_{P^{\prime}, k} \\ k \geq-\log _{2} \ell\left(P^{\prime}\right)}} T_{Q}\right) .
$$

Applying this with $K+n>J, J>n$, and $|Q|=\left|Q^{\prime}\right| \frac{|Q|}{\left|Q^{\prime}\right|}$ to the last inequality, it follows that

$$
\begin{aligned}
& |P|^{1-1 / p} E_{12} \\
\leq & C\left\{\sup _{P^{\prime} \in \mathscr{Q}(w)}\left|P^{\prime}\right|^{1-1 / p} \sup _{\substack{Q \in \mathscr{Q}_{P^{\prime}, k} \\
k \geq-\log _{2} \ell\left(P^{\prime}\right)}} T_{Q}\left[\sum_{i=1}^{\infty} \sum_{k \geq j} 2^{-K(k-j)} 2^{-\left(i-k_{0}\right) J} 2^{-j(J-n)} 2^{-j n} 2^{-(k-j) n} 2^{i n}\right]\right\} \\
\leq & C \sup _{P^{\prime} \in \mathscr{Q}(w)}\left|P^{\prime}\right|^{1-1 / p} \sup _{\substack{Q \in \mathscr{Q}_{P^{\prime}, k} \\
k \geq-\log _{2} \ell\left(P^{\prime}\right)}} \inf _{u \in Q}\left|\widetilde{\varphi}_{k} * f(u)\right| .
\end{aligned}
$$

To estimate $E_{2}$, for $i \in \mathbb{N}$ and $k<j$, set $G_{i, k}$ as $(3.3)$. Then $\left|x_{Q}-x_{P}\right| \approx 2^{i-k_{0}}$ for $Q \in G_{i, k}$ and

$$
E_{2}=\sup _{\substack{Q^{\prime} \in \mathcal{Q}_{P, j} \\ j \geq-\log _{2} \ell(P)}} \sum_{k<j} \sum_{i=1}^{\infty} \sum_{Q \in G_{i, k}} \frac{2^{-K|j-k|}}{|Q|^{1-1 / p}} \frac{2^{-k(J-n)}}{\left(2^{-k}+\left|x_{Q^{\prime}}-x_{Q}\right|\right)^{J}}|Q||Q|^{1-1 / p} T_{Q}
$$

Since $J>n$ and $K+n>J$, there are at most $2^{\left(i+k-k_{0}\right) n}$ dyadic cubes contained in $G_{i, k}$ and

$$
\begin{gathered}
|Q|^{1-1 / p} T_{Q} \leq \sup _{P^{\prime} \in \mathscr{Q}(w)}\left|P^{\prime}\right|^{1-1 / p} \sup _{\substack{R \in \mathscr{Q}_{P^{\prime}, m} \\
m \geq-\log _{2} \ell\left(P^{\prime}\right)}} T_{R}, \\
\leq C\left\{\sup _{P^{\prime} \in \mathscr{Q}(w)}\left|P^{\prime}\right|^{1-1 / p} \sup _{\substack{Q \in \mathscr{Q}_{P^{\prime}, m} \\
m \geq-\log _{2} \ell\left(P^{\prime}\right)}} T_{Q}\left[\sum_{k<j} \sum_{i=1}^{\infty} 2^{-K(j-k)} 2^{-\left(i-k_{0}\right) J} 2^{-k(J-n)} 2^{-k n} 2^{\left(i+k-k_{0}\right) n}\right]\right\} \\
\leq C \sup _{P^{\prime} \in \mathscr{Q}(w)}\left|P^{\prime}\right|^{1-1 / p} \sup _{\substack{Q \in \mathscr{Q}_{P^{\prime}, m} \\
m \geq-\log _{2} \ell\left(P^{\prime}\right)}} \inf _{u \in Q}\left|\widetilde{\varphi}_{m} * f(u)\right| .
\end{gathered}
$$

Combining the estimates of $E_{1}$ and $E_{2}$, we prove the theorem.

Theorem 3.3 (Plancherel-Pôlya inequality for $q \leq 1$ ). Let $\varphi, \phi \in \mathscr{S}$ satisfy (1.1). For $\alpha \in \mathbb{R}, 0<p \leq 1, q \leq 1$ and a weight $w$ with doubling exponent $\beta$, if $f \in \mathscr{S}^{\prime} / \mathscr{P}$ satisfies

$$
\sup _{P \in \mathscr{Q}(w)}\left\{|P|^{-q\left(1 / p-1 / q^{\prime}\right)} \sum_{k=-\log _{2} \ell(P)}^{\infty} \sum_{Q \in \mathscr{Q}_{P, k}}\left(2^{k \alpha} \sup _{u \in Q}\left|\widetilde{\varphi}_{k} * f(u)\right|\right)^{q} w(Q)\right\}^{1 / q}<\infty
$$


then

$$
\begin{aligned}
& \sup _{P \in \mathscr{Q}(w)}\left\{|P|^{-q\left(1 / p-1 / q^{\prime}\right)} \sum_{k=-\log _{2} \ell(P)}^{\infty} \sum_{Q \in \mathscr{Q}_{P, k}}\left(2^{k \alpha} \sup _{u \in Q}\left|\widetilde{\phi}_{k} * f(u)\right|\right)^{q} w(Q)\right\}^{1 / q} \\
\approx & \sup _{P \in \mathscr{Q}(w)}\left\{|P|^{-q\left(1 / p-1 / q^{\prime}\right)} \sum_{k=-\log _{2} \ell(P)}^{\infty} \sum_{Q \in \mathscr{Q}_{P, k}}\left(2^{k \alpha} \inf _{u \in Q}\left|\widetilde{\varphi}_{k} * f(u)\right|\right)^{q} w(Q)\right\}^{1 / q} .
\end{aligned}
$$

Proof. Without loss of generality, we may assume $\alpha=0$. By a similar argument as the proofs of Theorems 1.3 and 3.2 we have

$$
\sup _{u \in Q^{\prime}}\left|\widetilde{\phi}_{j} * f(u)\right| \leq C \sum_{k \in \mathbb{Z}} \sum_{Q \in \mathscr{Q}_{k}}\left(2^{-K|j-k|}|Q| \frac{2^{-(j \wedge k)(J-n)}}{\left(2^{-(j \wedge k)(J-n)}+\left|x_{Q^{\prime}}-x_{Q}\right|\right)^{J}}\right)^{q} T_{Q},
$$

where $J>\beta / q+n / q, K>(\beta / q-n) \vee(J-n / q-\beta / q)$ and $T_{Q}$ is as set out in (3.1).

Given a dyadic cube $P$ with $\ell(P)=2^{-k_{0}}$, the above estimates yield

$$
\begin{aligned}
& \sum_{j=k_{0}}^{\infty} \sum_{Q^{\prime} \in \mathscr{Q}_{P, j}}\left(\sup _{u \in Q^{\prime}}\left|\widetilde{\phi}_{j} * f(u)\right|\right)^{q} w\left(Q^{\prime}\right) \\
\leq & C \sum_{j=k_{0}}^{\infty} \sum_{Q^{\prime} \in \mathscr{Q}_{P, j}} \sum_{k \in \mathbb{Z}} \sum_{Q \in \mathscr{Q}_{k}}\left(2^{-K|j-k|} \frac{2^{-(j \wedge k)(J-n)}}{\left(2^{-(j \wedge k)}+\left|x_{Q^{\prime}}-x_{Q}\right|\right)^{J}}|Q|\right)^{q} \frac{w\left(Q^{\prime}\right)}{w(Q)} T_{Q} w(Q) \\
:= & C E_{1}+C E_{2},
\end{aligned}
$$

where

$$
E_{1}=\sum_{j=k_{0}}^{\infty} \sum_{Q^{\prime} \in \mathscr{Q}_{P, j}} \sum_{k \geq j} \sum_{Q \in \mathscr{Q}_{k}}\left(2^{-K|j-k|} \frac{2^{-(j \wedge k)(J-n)}}{\left(2^{-(j \wedge k)}+\left|x_{Q^{\prime}}-x_{Q}\right|\right)^{J}}|Q|\right)^{q} \frac{w\left(Q^{\prime}\right)}{w(Q)} T_{Q} w(Q)
$$

and

$$
E_{2}=\sum_{j=k_{0}}^{\infty} \sum_{Q^{\prime} \in \mathscr{Q}_{P, j}} \sum_{k<j} \sum_{Q \in \mathscr{Q}_{k}}\left(2^{-K|j-k|} \frac{2^{-(j \wedge k)(J-n)}}{\left(2^{-(j \wedge k)}+\left|x_{Q^{\prime}}-x_{Q}\right|\right)^{J}}|Q|\right)^{q} \frac{w\left(Q^{\prime}\right)}{w(Q)} T_{Q} w(Q) .
$$

Then $E_{1}$ can be further decomposed as

$$
\begin{aligned}
E_{1}= & \sum_{j=k_{0}}^{\infty} \sum_{Q^{\prime} \in \mathscr{Q}_{P, j}} \sum_{k \geq j} \sum_{Q \in \mathscr{Q}_{3 P, k}}\left(2^{-K|j-k|} \frac{2^{-(j \wedge k)(J-n)}}{\left(2^{-(j \wedge k)}+\left|x_{Q^{\prime}}-x_{Q}\right|\right)^{J}}|Q|\right)^{q} \frac{w\left(Q^{\prime}\right)}{w(Q)} T_{Q} w(Q) \\
& +\sum_{j=k_{0}}^{\infty} \sum_{Q^{\prime} \in \mathscr{Q}_{P, j}} \sum_{\substack { k \geq j \\
\begin{subarray}{c}{Q \cap 3 P=\emptyset \\
Q \in \mathscr{Q}_{k}{ k \geq j \\
\begin{subarray} { c } { Q \cap 3 P = \emptyset \\
Q \in \mathscr { Q } _ { k } } }\end{subarray}}\left(2^{-K|j-k|} \frac{2^{-(j \wedge k)(J-n)}}{\left(2^{-(j \wedge k)}+\left|x_{Q^{\prime}}-x_{Q}\right|\right)^{J}}|Q|\right)^{q} \frac{w\left(Q^{\prime}\right)}{w(Q)} T_{Q} w(Q) \\
:= & E_{11}+E_{12} .
\end{aligned}
$$


There are $3^{n}$ dyadic cubes in $3 P$ with the same side length as $P$, so if $P^{\prime} \subseteq 3 P$ then $\left|P^{\prime}\right|=|P|$ and

$$
\sum_{Q \in \mathscr{Q}_{3 P, k}} T_{Q} w(Q) \leq 3^{n} \sup _{\substack{P^{\prime} \subseteq 3 P \\ \ell\left(P^{\prime}\right)=\ell(P)}} \sum_{Q \in \mathscr{Q}_{P^{\prime}, k}} T_{Q} w(Q) .
$$

Let $J>\beta / q, k \geq j$ and $w$ be a weight with doubling exponent $\beta$. By Lemma 3.1 we have

$$
\int_{\mathbb{R}^{n}}\left(1+\frac{\left|x-x_{Q}\right|}{2^{-j}}\right)^{-J} w(x) d x \leq c_{\beta} 2^{(k-j) \beta} w(Q),
$$

and so

$$
\begin{aligned}
& \sum_{j=k_{0}}^{\infty} \sum_{Q^{\prime} \in \mathscr{Q}_{P, j}}\left(2^{-K|j-k|} \frac{2^{-(j \wedge k)(J-n)}}{\left(2^{-(j \wedge k)}+\left|x_{Q^{\prime}}-x_{Q}\right|\right)^{J}}|Q|\right)^{q} w\left(Q^{\prime}\right) \\
\leq & C \sum_{j=k_{0}}^{\infty} 2^{-K q(k-j)} 2^{j n q} 2^{-k n q} \int_{\mathbb{R}^{n}}\left(1+\frac{\left|x-x_{Q}\right|}{2^{-j}}\right)^{-J q} w(x) d x \\
\leq & C w(Q) .
\end{aligned}
$$

Hence

$$
\begin{aligned}
& |P|^{-q\left(1 / p-1 / q^{\prime}\right)} E_{11} \\
& \leq C|P|^{-q\left(1 / p-1 / q^{\prime}\right)} \sum_{j=k_{0}}^{\infty} \sum_{Q^{\prime} \in \mathscr{Q}_{P, j}} \sum_{k \geq j} \sum_{Q \in \mathscr{Q}_{3 P, k}}\left(2^{-K|j-k|} \frac{2^{-(j \wedge k)(J-n)}}{\left(2^{-(j \wedge k)}+\left|x_{Q^{\prime}}-x_{P}\right|\right)^{J}}|Q|\right)^{q} \\
& \times \frac{w\left(Q^{\prime}\right)}{w(Q)} T_{Q} w(Q) \\
& \leq C \sup _{P^{\prime} \in \mathscr{Q}(w)}\left|P^{\prime}\right|^{-q\left(1 / p-1 / q^{\prime}\right)} \sum_{k=-\log _{2} \ell\left(P^{\prime}\right)}^{\infty} \sum_{Q \in \mathscr{Q}_{P^{\prime}, k}} \inf _{u \in Q}\left|\widetilde{\varphi}_{k} * f(u)\right|^{q} w(Q) .
\end{aligned}
$$

Next, we decompose the set of dyadic cubes $\{Q: Q \cap 3 P=\emptyset, \ell(Q)=\ell(P)\}$ into $\left\{B_{i}\right\}_{i \in \mathbb{N}}$ as 3.2 . Then we obtain

$$
\begin{aligned}
& |P|^{-q\left(1 / p-1 / q^{\prime}\right)} E_{12} \\
\leq & C \sum_{i=1}^{\infty} \sum_{P^{\prime} \in B_{i}}\left|P^{\prime}\right|^{-q\left(1 / p-1 / q^{\prime}\right)} \\
& \times \sum_{j=k_{0}}^{\infty} \sum_{Q^{\prime} \in \mathscr{Q}_{P, j}} \sum_{\substack { k \geq j \\
\begin{subarray}{c}{Q \in \mathscr{Q}_{P^{\prime}, k} \\
P^{\prime} \in B_{i}{ k \geq j \\
\begin{subarray} { c } { Q \in \mathscr { Q } _ { P ^ { \prime } , k } \\
P ^ { \prime } \in B _ { i } } }\end{subarray}}\left(2^{-K|j-k|} \frac{2^{-j(J-n)}}{\left(2^{-j}+\left|x_{Q^{\prime}}-x_{P}\right|\right)^{J}}|Q|\right)^{q} \frac{w\left(Q^{\prime}\right)}{w(Q)} T_{Q} w(Q) .
\end{aligned}
$$

Since $w\left(Q^{\prime}\right) / w(Q) \leq C 2^{\beta\left(k-k_{0}+i\right)}$ and $\left\|x_{P^{\prime}}-x_{P}\right\| \approx 2^{i-k_{0}}$ for $P^{\prime} \in B_{i}$, the right-hand side 
of the inequality is dominated by

$$
\begin{aligned}
& C \sum_{i=1}^{\infty} \sum_{P^{\prime} \in B_{i}} \sum_{j=k_{0}}^{\infty} \sum_{Q^{\prime} \in \mathscr{Q}_{P, j}} \sum_{k \geq j} \sum_{\substack{Q \in \mathscr{Q}_{P^{\prime}, k} \\
P^{\prime} \in B_{i}}} 2^{\beta\left(k-k_{0}+i\right)} 2^{-K q(k-j)} 2^{-q\left(i-k_{0}\right) J} 2^{-j(J-n) q}|Q|^{q} \\
& \times\left(\sup _{P^{\prime} \in \mathscr{Q}(w)}\left|P^{\prime}\right|^{-q\left(1 / p-1 / q^{\prime}\right)} \sum_{k \geq k_{0}} \sum_{Q \in \mathscr{Q}_{P^{\prime}, k}} T_{Q} w(Q)\right) .
\end{aligned}
$$

Because there are at most $2^{(i+2) n}$ dyadic cubes in $B_{i}, J>\beta / q+n, K+n>\beta q$ and $|Q|^{q}=\left|Q^{\prime}\right|^{q} \frac{|Q|^{q}}{\left|Q^{\prime}\right|^{q}}$,

$$
\begin{aligned}
& |P|^{-q\left(1 / p-1 / q^{\prime}\right)} E_{12} \\
\leq & C\left\{\sup _{P^{\prime} \in \mathscr{Q}}\left|P^{\prime}\right|^{-q\left(1 / p-1 / q^{\prime}\right)} \sum_{k \geq k_{0}} \sum_{Q \in \mathscr{Q}_{P^{\prime}, k}} T_{Q} w(Q)\right\} \\
& \times\left[\sum_{i=1}^{\infty} \sum_{j=k_{0}}^{\infty} \sum_{k \geq j} 2^{\beta\left(k-k_{0}+i\right)} 2^{-K q(k-j)} 2^{-q\left(i-k_{0}\right) J} 2^{-q(J-n) j} 2^{-k_{0} n q} 2^{(j-k) n q} 2^{i n}\right] \\
\leq & C \sup _{P^{\prime} \in \mathscr{Q}(w)}\left|P^{\prime}\right|^{-q\left(1 / p-1 / q^{\prime}\right)} \sum_{k=-\log _{2} \ell\left(P^{\prime}\right)}^{\infty} \sum_{Q \in \mathscr{Q}_{P^{\prime}, k}} \inf _{u \in Q}\left|\widetilde{\varphi}_{k} * f(u)\right|^{q} w(Q) .
\end{aligned}
$$

To estimate $E_{2}$, for $i \in \mathbb{N}$ and $k<k_{0}$, set $G_{i, k}$ as 3.3$)$. Then $\left|x_{Q}-x_{P}\right| \approx 2^{i-k_{0}}$ for $Q \in G_{i, k}$ and

$$
\begin{gathered}
E_{2}=\sum_{j=k_{0}}^{\infty} \sum_{Q^{\prime} \in \mathscr{Q}_{P, j}} \sum_{k<j} \sum_{i=1}^{\infty} \sum_{Q \in G_{i, k}} \frac{2^{-K|j-k| q}}{|Q|^{-q\left(1 / p-1 / q^{\prime}\right)}}\left(\frac{2^{-k(J-n)}}{\left(2^{-k}+\left|x_{Q^{\prime}}-x_{Q}\right|^{J}\right.}\right)^{q} \frac{w\left(Q^{\prime}\right)}{w(Q)}|Q|^{q} \\
\times|Q|^{-q\left(1 / p-1 / q^{\prime}\right)} T_{Q} w(Q) .
\end{gathered}
$$

Since $J>\beta / q+n / q$ and $K>J-n / q-\beta / q$, there are at most $2^{\left(i+k-k_{0}\right) n}$ dyadic cubes contained in $G_{i, k}$ and

$$
\begin{aligned}
& |Q|^{-q\left(1 / p-1 / q^{\prime}\right)} T_{Q} w(Q) \leq \sup _{P^{\prime} \in \mathscr{Q}(w)}\left|P^{\prime}\right|^{-q\left(1 / p-1 / q^{\prime}\right)} \sum_{m \geq-\log _{2} \ell\left(P^{\prime}\right)} \sum_{R \in \mathscr{Q}_{P^{\prime}, m}} T_{R} w(R), \\
& |Q|^{-q\left(1 / p-1 / q^{\prime}\right)} E_{2} \\
& \leq C\left\{\sup _{P^{\prime} \in \mathscr{Q}(w)}\left|P^{\prime}\right|^{-q\left(1 / p-1 / q^{\prime}\right)} \sum_{m \geq-\log _{2} \ell\left(P^{\prime}\right)} \sum_{Q \in \mathscr{Q}_{P^{\prime}, m}} T_{Q} w(Q)\right\} \\
& \quad \times\left[\sum_{j=k_{0}}^{\infty} \sum_{k<j} \sum_{i=1}^{\infty} 2^{-K q(j-k)} 2^{-q\left(i-k_{0}\right) J} 2^{-k q(J-n)} 2^{\beta\left(k-k_{0}+i\right)} 2^{-k n q} 2^{\left(i+k-k_{0}\right) n}\right] \\
& \leq C \sup _{P^{\prime} \in \mathscr{Q}(w)}\left|P^{\prime}\right|^{-q\left(1 / p-1 / q^{\prime}\right)} \sum_{m \geq-\log _{2} \ell\left(P^{\prime}\right)} \sum_{Q \in \mathscr{Q}_{P^{\prime}, m}} \inf _{u \in Q}\left|\widetilde{\varphi}_{m} * f(u)\right|^{q} w(Q) .
\end{aligned}
$$


By those estimates above, we have the desired result.

Remark 3.4. The classical Plancherel-Pôlya inequality [14 concludes that if $\left\{x_{k}\right\}$ is an appropriate set of points in $\mathbb{R}^{n}$, e.g., lattice points, where the length of the mesh is sufficiently small, then

$$
\left(\sum_{k=1}^{\infty}\left|f\left(x_{k}\right)\right|^{p}\right)^{1 / p} \approx\|f\|_{p}
$$

for all $0<p \leq \infty$, with a modification if $p=\infty$.

\section{Norm equivalence}

In this section, we study the norm equivalence between $\dot{C} M O_{p, w}^{\alpha, q}$ and $\dot{c}_{p, w}^{\alpha, q}$. Suppose that $w \in A_{\infty}$ and let $r_{0}=\inf \left\{r: w \in A_{r}\right\}$. For $\alpha \in \mathbb{R}$ and $0<p, q \leq \infty$, let $H=$ $\max \left\{n, n r_{0} / p, n / q\right\}$. We say that a matrix $A=\left\{a_{Q P}\right\}_{Q, P}$ is $(\alpha, p, q)$-almost diagonal, denoted by $A \in \operatorname{ad}_{p}^{\alpha, q}(w)$ if there exists $\varepsilon>0$ such that

$$
\sup _{Q, P} \frac{\left|a_{Q P}\right|}{\omega_{Q P(\varepsilon)}}<\infty
$$

where

$$
\begin{aligned}
\omega_{Q P}(\varepsilon)= & \left(\frac{\ell(Q)}{\ell(P)}\right)^{\alpha}\left(1+\frac{\left|x_{Q}-x_{P}\right|}{\max (\ell(P), \ell(Q))}\right)^{-H-\varepsilon} \\
& \times \min \left\{\left(\frac{\ell(Q)}{\ell(P)}\right)^{(n+\varepsilon) / 2},\left(\frac{\ell(P)}{\ell(Q)}\right)^{(n+\varepsilon) / 2+H-n}\right\} .
\end{aligned}
$$

Lemma 4.1. Let $\alpha \in \mathbb{R}, 0<p<\infty, 0<q \leq \infty$ and $w \in A_{\infty}$. If $A$ is $(\alpha, p, q)$-almost diagonal, then $A$ is bounded on $\dot{f}_{p, w}^{\alpha, q}$.

Proof. We may assume $\alpha=0$, since the case implies the general case, and set $r=$ $\min \left(p / r_{0}, q\right)$. We shall consider the case $r>1$ first. Let $A$ be an $(0, p, q)$ almost diagonal operator on $\dot{f}_{p, w}^{0, q}$ with matrix $\left\{a_{Q, P}\right\}_{Q, P}$. We decompose the matrix operator $A$ as the sum of $A=A_{u}+A_{l}$, namely for $Q \in \mathscr{Q}(w)$

$$
\left(A_{u} s\right)_{Q}=\sum_{\substack{P \in \mathscr{Q}(w) \\ \ell(P) \geq \ell(Q)}} a_{Q P} s_{P} \quad \text { and } \quad\left(A_{l} s\right)_{Q}=\sum_{\substack{P \in \mathscr{Q}(w) \\ \ell(P)<\ell(Q)}} a_{Q P} s_{P}
$$

for $s=\left\{s_{P}\right\} \in \dot{f}_{p, w}^{0, q}$. According to Lemma A.2 in [6], with $\lambda=H+\varepsilon$ and $a=r=1$,

$$
\begin{aligned}
\left|\left(A_{u} s\right)_{Q}\right| & \leq C \sum_{\substack{P \in \mathscr{Q}(w) \\
\ell(P) \geq \ell(Q)}}\left(1+\frac{\left|x_{Q}-x_{P}\right|}{\ell(P)}\right)^{-H-\varepsilon}\left(\frac{\ell(Q)}{\ell(P)}\right)^{(n+\varepsilon) / 2}\left|s_{P}\right| \\
& \leq C \sum_{j \leq k} 2^{(j-k)(n+\varepsilon) / 2} M\left(\sum_{P \in \mathscr{Q}_{j}(w)}\left|s_{P}\right| \chi_{P}\right)(x) \quad \text { for } x \in Q
\end{aligned}
$$


when $\ell(Q)=2^{-k}$.

Hence, since $|Q|^{-1 / 2}=2^{(k-j) n / 2}|P|^{-1 / 2}$ if $\ell(P)=2^{-j}$,

$$
\begin{aligned}
\left\|A_{u} \boldsymbol{s}\right\|_{f_{p, w}^{0, q}} & \leq C\left\|\left(\sum_{k \in \mathbb{Z}}\left(\sum_{j \leq k} 2^{(j-k)(n+\varepsilon) / 2} M\left(\sum_{P \in \mathscr{Q}_{j}(w)}|P|^{-1 / 2}\left|s_{P}\right| \chi_{P}\right)(x)\right)^{q}\right)^{1 / q}\right\|_{L^{p}(w)} \\
& \leq C\left\|\left(\sum_{j \in \mathbb{Z}}\left(M\left(\sum_{P \in \mathscr{Q}_{j}(w)}|P|^{-1 / 2}\left|s_{P}\right| \chi_{P}\right)(x)\right)^{q}\right)^{1 / q}\right\|_{L^{p}(w)}
\end{aligned}
$$

by Minkowski's inequality. Applying the weighted version of Fefferman-Stein vector-valued maximal inequality which was characterized by Andersen and John [1, we find that

$$
\left\|A_{u} s\right\|_{\dot{f}_{p, w}^{0, q}} \leq C\|s\|_{\dot{f}_{p, w}^{0, q}}
$$

since $q>1, p>r_{0}$ and $w \in A_{p}$.

Next, consider the case for the matrix operator $A_{l}$. Observe that if $\ell(P) \leq \ell(Q)$, then

$$
\frac{\ell(P)}{\ell(Q)}\left(1+\frac{\left|x_{Q}-x_{P}\right|}{\ell(Q)}\right) \leq 1+\frac{\left|x_{Q}-x_{P}\right|}{\ell(Q)}
$$

Thus

$$
\begin{aligned}
\left(1+\frac{\left|x_{Q}-x_{P}\right|}{\ell(Q)}\right)^{-H-\varepsilon} & \leq\left(1+\frac{\left|x_{Q}-x_{P}\right|}{\ell(Q)}\right)^{-H-\varepsilon / 4} \\
& \leq\left(1+\frac{\left|x_{Q}-x_{P}\right|}{\ell(Q)}\right)^{-H-\varepsilon / 4}\left(\frac{\ell(P)}{\ell(Q)}\right)^{-H-\varepsilon / 4},
\end{aligned}
$$

and, for $Q \in \mathscr{Q}_{k}(w)$,

$$
\begin{aligned}
\left|\left(A_{l} s\right)_{Q}\right| & \leq C \sum_{\substack{P \in \mathscr{Q}(w) \\
\ell(P)<\ell(Q)}}\left(1+\frac{\left|x_{Q}-x_{P}\right|}{\ell(Q)}\right)^{-H-\varepsilon}\left(\frac{\ell(P)}{\ell(Q)}\right)^{(n+\varepsilon) / 2+H-n}\left|s_{P}\right| \\
& \leq C \sum_{\substack{P \in \mathscr{Q}(w) \\
\ell(P)<\ell(Q)}}\left(1+\frac{\left|x_{Q}-x_{P}\right|}{\ell(Q)}\right)^{-H-\varepsilon / 4}\left(\frac{\ell(P)}{\ell(Q)}\right)^{\varepsilon / 4-n / 2}\left|s_{P}\right| \\
& \leq C \sum_{j>k} 2^{(k-j)(-n / 2+\varepsilon / 4)} M\left(\sum_{P \in \mathscr{Q}_{j}(w)}\left|s_{P}\right| \chi_{P}\right)(x) \text { for } x \in Q .
\end{aligned}
$$


Hence, since $|Q|^{-1 / 2}=2^{(k-j) n / 2}|P|^{-1 / 2}$ if $\ell(P)=2^{-j}$,

$$
\begin{aligned}
\left\|A_{l} s\right\| & \leq C\left\|\left(\sum_{k \in \mathbb{Z}}\left(\sum_{j>k} 2^{(k-j) \varepsilon / 4} M\left(\sum_{P \in \mathscr{Q}_{j}(w)}|P|^{-1 / 2}\left|s_{P}\right| \chi_{P}\right)(x)\right)^{q}\right)^{1 / q}\right\|_{L^{p}(w)} \\
& \leq C\left\|\left(\sum_{j \in \mathbb{Z}}\left(M\left(\sum_{P \in \mathscr{Q}_{j}(w)}|P|^{-1 / 2}\left|s_{P}\right| \chi_{P}\right)(x)\right)^{q}\right)^{1 / q}\right\|_{L^{p}(w)}
\end{aligned}
$$

by Minkowski's inequality. Applying Proposition 2.4, we find that

$$
\left\|A_{l} s\right\|_{\dot{f}_{p, w}^{0, q}} \leq C\|s\|_{\dot{f}_{p, w}^{0, q}}
$$

since $p, q>1, p>r_{0}$ and $w \in A_{p}$.

The case $r \leq 1$ and $q<\infty$ is in fact a consequence of the case $r>1$. We pick an $\widetilde{r}<r$ so close to $r$ that 4.1$)$ is still satisfied with $r=\min \left(p / r_{0}, q\right)$ replaced by $\widetilde{r}$. This means that $p / \widetilde{r}>1$ and $q / \widetilde{r}>1$, and that the matrix $\widetilde{A}=\left\{\widetilde{a}_{Q P}\right\}:=\left\{\left|a_{Q P}\right|^{\widetilde{r}}(|Q| /|P|)^{1 / 2-\widetilde{r} / 2}\right\}$ satisfies 4.2 for a different value of $\varepsilon$. Define $\boldsymbol{t}=\left\{t_{Q}\right\}_{Q}$ by $t_{Q}=|Q|^{1 / 2-\widetilde{r} / 2}\left|s_{Q}\right|^{\widetilde{r}}$. Then $\|\boldsymbol{t}\|_{\dot{f}_{p / \widetilde{r}, w}^{0, q / \widetilde{r}}}^{1 / \widetilde{r}}=\|s\|_{f_{p, w}^{0, q}}$. By the $\widetilde{r}$-triangle inequality, we have

$$
\left|(A s)_{Q}\right| \leq\left(\sum_{P \in \mathscr{Q}(w)}\left|a_{Q P}\right|^{\widetilde{r}}\left|s_{P}\right|^{\widetilde{r}}\right)^{1 / \widetilde{r}}
$$

Hence, $\|A s\|_{\dot{f}_{p, w}^{0, q}} \leq\|\widetilde{A} \boldsymbol{t}\|_{\dot{f}_{p / \widetilde{r}, w}^{0, q / \widetilde{r}}}^{1 / \widetilde{r}}$. Therefore the boundedness of $A$ on $\dot{f}_{p, w}^{0, q}$ follows from the boundedness of $\widetilde{A}$ on $\dot{f}_{p / \widetilde{r}, w}^{0, q / \widetilde{r}}$. By duality, the case $q=\infty$ and $p>1$ follows from the result of $q=1$ which we have just obtained. Finally, for $p \leq 1$ and $q=\infty$, we reduce to the case $p>1$ as before.

Next let us consider the boundedness of almost diagonal operators acting on weighted Carleson measure sequence spaces $\dot{c}_{p, w}^{\alpha, q}$. In particular, consider the boundedness of almost diagonal operators acting on $\dot{f}_{\infty, w}^{\alpha, q}$. Under this situation, we always assume $H=$ $\max \left\{n, n r_{0} / p\right\}$ in the definition of almost diagonality.

Lemma 4.2. For $\alpha \in \mathbb{R}, 0<p \leq 1,0<q \leq \infty$ and $w \in A_{\infty}$, an $\left(\alpha+n q\left(1 / p-1 / q^{\prime}\right), p, q\right)$ almost diagonal matrix is bounded on $\dot{c}_{p, w}^{\alpha, q}$.

Proof. We may assume $\alpha=0$, since the case implies the general case. Let $A=\left\{a_{Q P}\right\}_{Q, P}$ be an $\left(n q\left(1 / p-1 / q^{\prime}\right), p, q\right)$-almost diagonal matrix. Write $A=A_{u}+A_{l}$ with

$$
\left(A_{u} s\right)_{Q}=\sum_{\substack{P \in \mathscr{Q}(w) \\ \ell(P) \geq \ell(Q)}} a_{Q P} s_{P} \text { and } \quad\left(A_{l} s\right)_{Q}=\sum_{\substack{P \in \mathscr{Q}(w) \\ \ell(P)<\ell(Q)}} a_{Q P} s_{P}
$$


for $s=\left\{s_{P}\right\} \in \dot{c}_{p, w}^{0, q}$. Set $r=n q\left(1 / p-1 / q^{\prime}\right)$. If $\ell(Q)=2^{-k}$, then

$$
\begin{aligned}
\left|\left(A_{u} s\right)_{Q}\right| & \leq C \sum_{\substack{P \in \mathscr{Q}(w) \\
\ell(P) \geq \ell(Q)}}\left(\frac{\ell(Q)}{\ell(P)}\right)^{r}\left(1+\frac{\left|x_{Q}-x_{P}\right|}{\max (\ell(P), \ell(Q))}\right)^{-H-\varepsilon}\left(\frac{\ell(Q)}{\ell(P)}\right)^{(n+\varepsilon) / 2}\left|s_{P}\right| \\
& \leq C \sum_{j=-\infty}^{k} 2^{(j-k)[r+(n+\varepsilon) / 2]} M\left(\sum_{P \in \mathscr{Q}_{j}(w)}\left|s_{P}\right| \chi_{P}\right)(x) \quad \text { for } x \in Q .
\end{aligned}
$$

Hence, since $|Q|^{-1 / 2}=2^{(k-j) n / 2}|P|^{-1 / 2}$ if $\ell(P)=2^{-j}$,

$$
|Q|^{-1 / 2}\left|\left(A_{u} s\right)_{Q}\right| \chi_{Q}(x) \leq C \sum_{j=-\infty}^{k} 2^{(j-k)(r+\varepsilon / 2)} M\left(\sum_{P \in \mathscr{Q}_{j}(w)}|P|^{-1 / 2}\left|s_{P}\right| \chi_{P}\right)(x) \chi_{Q}(x)
$$

and, by Hölder's inequality,

$$
\begin{aligned}
&\left\|A_{u} s\right\|_{\dot{c}_{p, w}^{0, q}} \leq C \sup _{R \in \mathscr{Q}(w)}\left\{|R|^{-r / n} \int_{R} \sum_{k \geq-\log _{2} \ell(R)} \sum_{Q \in \mathscr{Q}_{R, k}(w)} \sum_{j=-\infty}^{k} 2^{(j-k)(r+\varepsilon / 2)}\right. \\
& \times {\left.\left[M\left(\sum_{P \in \mathscr{Q}_{j}(w)}|P|^{-1 / 2}\left|s_{P}\right| \chi_{P}\right)(x)\right]^{q} \chi_{Q}(x) w(x) d x\right\}^{1 / q} } \\
& \leq C \sup _{R \in \mathscr{Q}(w)}\left\{|R|^{-r / n} \int_{\mathbb{R}^{n}} \sum_{k \geq-\log _{2} \ell(R)} \sum_{j=-\infty}^{k} 2^{(j-k)(r+\varepsilon / 2)}\right. \\
&\left.\times\left[M\left(\sum_{P \in \mathscr{Q}_{j}(w)}|P|^{-1 / 2}\left|s_{P}\right| \chi_{P}\right)(x)\right]^{q} w(x) d x\right\}^{1 / q} .
\end{aligned}
$$

Note that for given $R$ with $\ell(R)=2^{-\delta}$,

$$
\begin{aligned}
& \sum_{k \geq-\log _{2} \ell(R)} \sum_{j=-\infty}^{k} 2^{(j-k)(r+\varepsilon / 2)}\left[M\left(\sum_{P \in \mathscr{Q}_{j}(w)}|P|^{-1 / 2}\left|s_{P}\right| \chi_{P}\right)(x)\right]^{q} \\
= & \sum_{j=-\infty}^{\delta-1} \sum_{k=\delta}^{\infty} 2^{(j-\delta)(r+\varepsilon / 2)} 2^{(\delta-k)(r+\varepsilon / 2)}\left[M\left(\sum_{P \in \mathscr{Q}_{j}(w)}|P|^{-1 / 2}\left|s_{P}\right| \chi_{P}\right)(x)\right]^{q} \\
& \left.+\sum_{j=\delta}^{\infty} \sum_{k=j}^{\infty} 2^{(j-k)(r+\varepsilon / 2)}\left[M\left(\sum_{P \in \mathscr{Q}_{j}(w)}|P|^{-1 / 2}\left|s_{P}\right| \chi_{P}\right)(x)\right]^{q}\right]^{q} \\
\leq & \left.C \sum_{j=-\infty}^{\delta-1} 2^{(j-\delta)(r+\varepsilon / 2)}\left[M\left(\sum_{P \in \mathscr{Q}_{j}(w)}|P|^{-1 / 2}\left|s_{P}\right| \chi_{P}\right)(x)\right]^{q}\right]^{q} \cdot\left(\left.x\right|_{P \in \mathscr{Q}_{j}(w)}|P|^{-1 / 2}\left|s_{P}\right| \chi_{P}\right)(x) \\
+ & C \sum_{j=\delta}^{\infty}\left[M \left(\sum_{\mid}[M\right.\right.
\end{aligned}
$$


Applying Proposition 2.4 if $P_{0}$ is the cube containing $R$ with $\ell\left(P_{0}\right)=2^{-j}$, then we get

$$
\begin{aligned}
& \quad|R|^{-r / n} \int_{R} \sum_{k \geq-\log _{2} \ell(R)} \sum_{j=-\infty}^{k} 2^{(j-k)(r+\varepsilon / 2)}\left[M\left(\sum_{P \in \mathscr{Q}_{j}(w)}|P|^{-1 / 2}\left|s_{P}\right| \chi_{P}\right)(x)\right]^{q} w(x) d x \\
& \leq C \sum_{j=-\infty}^{\delta-1} 2^{(j-\delta)(r+\varepsilon / 2)}\left(\frac{|R|}{\left|P_{0}\right|}\right)^{-r / n}\left|P_{0}\right|^{-r / n} \int_{P_{0}}\left(\left|P_{0}\right|^{-1 / 2}\left|s_{P_{0}}\right| \chi_{P_{0}}(x)\right)^{q} w(x) d x \\
& \quad+C|R|^{-r / n} \int_{R} \sum_{j \geq-\log _{2} \ell(R)}\left(\sum_{P \in \mathscr{Q}_{j}(w)}|P|^{-1 / 2}\left|s_{P}\right| \chi_{P}(x)\right)^{q} w(x) d x \\
& \leq C \sum_{j=-\infty}^{\delta-1} 2^{(j-\delta)(r+\varepsilon / 2)} 2^{(j-\delta) n(-r / n)}\left|P_{0}\right|^{-r / n} \int_{P_{0}}\left(\left|P_{0}\right|^{-1 / 2}\left|s_{P_{0}}\right| \chi_{P_{0}}(x)\right)^{q} w(x) d x \\
& \quad+C|R|^{-r / n} \int_{R} \sum_{j \geq-\log _{2} \ell(R)}\left(\sum_{P \in \mathscr{Q}_{j}(w)}|P|^{-1 / 2}\left|s_{P}\right| \chi_{P}(x)\right)^{q} w(x) d x,
\end{aligned}
$$

because $|R| /\left|P_{0}\right|=2^{(j-\delta) n}$. Hence

$$
\left\|A_{u} s\right\|_{\dot{c}_{p, w}^{0, q}} \leq C\|s\|_{\dot{c}_{p, w}^{0, q}} .
$$

A similar argument for $A_{l}$ yields $\left\|A_{l} s\right\|_{\dot{c}_{p, w}^{0, q}} \leq C\|s\|_{\dot{c}_{p, w}^{0, q}}$.

Finally, we can give a proof for Theorem 1.7 .

Proof of Theorem 1.7. For $0<q<\infty$ and $f \in \dot{C} M O_{p, w}^{\alpha, q}$, let $s:=\left\{s_{Q}\right\}_{Q}=S_{\varphi}(f)$. Then the $\varphi$-transform identity shows $f=\sum_{Q} s_{Q} \psi_{Q}$ and $\|f\|_{\dot{C} M O_{p, w}^{\alpha, q}}=\left\|S_{\varphi}(f)\right\|_{\dot{C}_{p, w}^{\alpha, q}}=\|s\|_{\dot{C}_{p, w}^{\alpha, q}}$. In particular, $\|f\|_{\dot{C} M O_{1, w}^{\alpha, q}}=\left\|S_{\varphi}(f)\right\|_{\dot{c}_{1, w}^{\alpha, q}}=\left\|S_{\varphi}(f)\right\|_{\dot{f}_{\infty, w}^{\alpha, q}} \approx\|f\|_{\dot{F}_{\infty, w}^{\alpha, q}}$. Furthermore, for $s \in \dot{c}_{p, w}^{\alpha, q}$

$$
\left\|T_{\psi}(s)\right\|_{\dot{C} M O_{p, w}^{\alpha, q}}=\left\|\sum_{P} s_{P} \psi_{P}\right\|_{\dot{C} M O_{p, w}^{\alpha, q}}=\left\|\left\{\left\langle\sum_{P} s_{P} \psi_{P}, \varphi_{Q}\right\rangle\right\}_{Q}\right\|_{\dot{c}_{p, w}^{\alpha, q}}=\|A s\|_{\dot{c}_{p, w}^{\alpha, q},}
$$

where $A:=\left\{\left\langle\psi_{P}, \varphi_{Q}\right\rangle\right\}_{Q, P}$ is $\left(\alpha+n q\left(1 / p-1 / q^{\prime}\right), p, q\right)$-almost diagonal (cf. Lemma 3.6 in [6]) and hence $A$ is bounded on $\dot{c}_{p, w}^{\alpha, q}$ by Lemma 4.2. Therefore, $S_{\varphi}$ is bounded from $\dot{C} M O_{p, w}^{\alpha, q}$ to $\dot{c}_{p, w}^{\alpha, q}$ and $T_{\varphi}$ is bounded from $\dot{c}_{p, w}^{\alpha, q}$ to $\dot{C} M O_{p, w}^{\alpha, q}$. We summarize that $T_{\psi} \circ$ $\left.S_{\varphi}\right|_{\dot{C} M O_{p, w}^{\alpha, q}}$ is also the identity on $\dot{C} M O_{p, w}^{\alpha, q}$.

\section{Acknowledgments}

The authors are grateful to the referees for many invaluable suggestions. It is a good question to extend main results in this article. Namely, can we release the condition $w \in A_{p}$ to $w \in A_{p}^{d y}$ to guarantee the boundedness of almost diagonal operators acting on $\dot{c}_{p, w}^{\alpha, q} ?$ 


\section{References}

[1] K. F. Andersen and R. T. John, Weighted inequalities for vector-valued maximal functions and singular integrals, Studia Math. 69 (1980/81), no. 1, 19-31.

[2] M. Bownik and K.-P. Ho, Atomic and molecular decompositions of anisotropic TriebelLizorkin spaces, Trans. Amer. Math. Soc. 358 (2006), no. 4, 1469-1510.

[3] H.-Q. Bui, M. Paluszyński and M. H. Taibleson, A maximal function characterization of weighted Besov-Lipschitz and Triebel-Lizorkin spaces, Studia Math. 119 (1996), no. 3, 219-246.

[4] _ Characterization of the Besov-Lipschitz and Triebel-Lizorkin spaces: The case $q<1$, J. Fourier Anal. Appl. 3 (1997), Special Issue, 837-846.

[5] H.-Q. Bui and M. H. Taibleson, The characterization of the Triebel-Lizorkin spaces for $p=\infty$, J. Fourier Anal. Appl. 6 (2000), no. 5, 537-550.

[6] M. Frazier and B. Jawerth, A discrete transform and decompositions of distribution spaces, J. Funct. Anal. 93 (1990), no. 1, 34-170.

[7] M. Izuki and Y. Sawano, Wavelet bases in the weighted Besov and Triebel-Lizorkin spaces with $A_{p}^{\text {loc }}$-weights, J. Approx. Theory. 161 (2009), no. 2, 656-673.

[8] G. Kyriazis, P. Petrushev and Y. Xu, Decomposition of weighted Triebel-Lizorkin and Besov spaces on the ball, Proc. Lond. Math. Soc. (3) 97 (2008), no. 2, 477-513.

[9] M.-Y. Lee, C.-C. Lin and Y.-C. Lin, A wavelet characterization for the dual of weighted Hardy spaces, Proc. Amer. Math. Soc. 137 (2009), no. 12, 4219-4225.

[10] C.-C. Lin and K. Wang, Generalized Carleson measure spaces and their applications, Abstr. Appl. Anal. 2012 (2012), Art. ID 879073, 26 pp.

[11] F. L. Nazarov and S. R. Treul', The hunt for a Bellman function: Applications to estimates for singular integral operators and to other classical problems of harmonic analysis, Algebra i Analiz 8 (1996), no. 5, 32-162; St. Petersburg Math. J. 8 (1997), no. 5, 721-824.

[12] S. Roudenko, Matrix-weighted Besov spaces, Trans. Amer. Math. Soc. 355 (2003), no. 1, 273-314.

[13] A. Torchinsky, Real-variable Methods in Harmonic Analysis, Pure and Applied Mathematics 123, Academic Press, Orlando, FL, 1986. 
[14] H. Triebel, Theory of Function Spaces, Monographs in Mathematics 78, Birkhäuser Verlag, Basel, 1983.

Hsun-Wu Liu and Kunchuan Wang

Department of Applied Mathematics, National Dong Hwa University, Hualien, Taiwan E-mail address: takeshi.liu@livemail.tw, kcwang@gms.ndhu.edu.tw 